\title{
The overall coefficient of the two-loop superstring amplitude using pure spinors
}

\author{
Humberto Gomez ${ }^{a}$ and Carlos R. Mafra ${ }^{b}$ \\ ${ }^{a}$ Instituto de Fúsica Teórica, UNESP - Universidade Estadual Paulista, \\ Caixa Postal 70532-2, 01156-970 São Paulo, SP, Brazil \\ ${ }^{b}$ Max-Planck-Institut für Gravitationsphysik, Albert-Einstein-Institut, \\ Am Mühlenberg 1, 14476 Potsdam, Germany \\ E-mail: humgomzu@ift.unesp.br, crmafra@aei.mpg.de
}

ABSTRACT: Using the results recently obtained for computing integrals over (non-minimal) pure spinor superspace, we compute the coefficient of the massless two-loop four-point amplitude from first principles. Contrasting with the mathematical difficulties in the RNS formalism where unknown normalizations of chiral determinant formulæ force the two-loop coefficient to be determined only indirectly through factorization, the computation in the pure spinor formalism can be smoothly carried out.

KEYwords: Superstrings and Heterotic Strings, Superspaces

ARXIV EPRINT: 1003.0678 


\section{Contents}

1 Introduction 1

2 The conventions 3

2.1 The normalization of zero-modes 8

2.2 On the normalization of the holomorphic 1-forms 9

3 Tree-level $\quad 9$

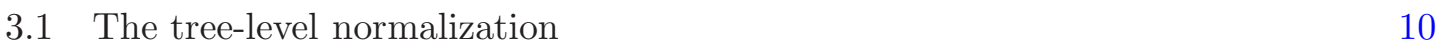

$\begin{array}{lll}4 \text { One-loop } & 11\end{array}$

5 Two-loop 13

6 Conclusions $\quad 15$

A Non-minimal two-loop kinematic factor $\quad 15$

B Period matrix parametrization of genus-two moduli space 20

\section{Introduction}

Scattering amplitudes led to the discovery of string theory more than 40 years ago. But after all these years, explicit results for higher-loop and/or higher-point amplitudes are relatively sparse. In fact, since the publication of the famous review by D'Hoker and Phong [1] in 1988, there has been a small number of new ten-dimensional scattering computations. Using either the RNS or GS formalisms, the extensions to our knowledge in higher loops [2] or higher points $[3-8]$ were limited to bosonic external states while the overall coefficients were not always under consideration. ${ }^{1}$

Since the discovery of the manifestly space-time supersymmetric pure spinor formalism [11-14] there has been progress in extending results of scattering amplitudes ${ }^{2}$ to the whole supermultiplet $[12,17-23]$ by using the pure spinor superspace [24] but explicit computations for genus higher than two are still missing though [25-27]. And the amplitudes in the pure spinor formalism were also computed up to the overall coefficients. That has changed since [28], where the precise normalizations for the pure spinor measures were determined and where it was also shown how to evaluate integrals in pure spinor space.

\footnotetext{
${ }^{1}$ There are however powerful approaches to discuss the coefficients which do not require direct tendimensional scattering computations $[9,10]$.

${ }^{2}$ The use of the pure spinor formalism however is not limited to scattering amplitudes only. For reviews, see $[15,16]$.
} 
So in this paper we use and extend the results of [28] to obtain the coefficient of the type IIB (and IIA [29, 30]) two-loop massless four-point amplitude from a first principles computation and for the whole supermultiplet. To achieve that we use pure spinor measures which present the feature of having simple forms for all genera, in deep contrast with the complicated superstring measure for the RNS formalism [31, 32]. As mentioned in [33], it is still an unsolved problem to find the precise normalizations for the chiral bosonization formulæ of [34]. Therefore the two-loop coefficient can not be obtained from a direct calculation in the RNS formalism. In fact, computing the amplitude up to the overall coefficient already required several years of effort which resulted in an impressive series of papers [2, 35-39], so the strategy adopted in [33] was to fix the two-loop coefficient indirectly by using factorization. So in this respect the calculations of this paper make it very clear how the pure spinor formalism can surpass the RNS limitations. But to present our results we have chosen to adopt the clear conventions of [33], which also eases the detection of any mismatches.

In section 2 the conventions and several pure spinor specific results are written down. Emphasis is made regarding the generality and simplicity of the pure spinor setup. The computations of the three- and four-point amplitudes at tree-level are performed in section 3 to show that the conventions of section 2 match the RNS ones of [33] such that $\mathcal{A}_{0}^{\mathrm{PS}}=\mathcal{A}_{0}^{\mathrm{RNS}}$, where

$$
\mathcal{A}_{0}^{\mathrm{PS}}=(2 \pi)^{10} \delta^{(10)}(k) \kappa^{4} e^{-2 \lambda}\left(\frac{\sqrt{2}}{2^{12} \pi^{6} \alpha^{\prime 5}}\right)\left(\frac{\alpha^{\prime}}{2}\right)^{8} K \bar{K} C(s, t, u)
$$

Then we use the very same machinery of the tree-level computation to obtain also the full supersymmetric one- and two-loop amplitudes - including their precise coefficients - in sections 4 and 5 ,

$$
\begin{aligned}
& \mathcal{A}_{1}^{\mathrm{PS}}=(2 \pi)^{10} \delta^{(10)}(k) \frac{\kappa^{4} K \bar{K}}{2^{9} \pi^{2} \alpha^{\prime 5}}\left(\frac{\alpha^{\prime}}{2}\right)^{8} \int_{\mathcal{M}_{1}} \frac{d^{2} \tau}{\tau_{2}^{5}} \prod_{i=2}^{4} \int d^{2} z_{i} \prod_{i<j}^{4} F_{1}\left(z_{i}, z_{j}\right)^{\alpha k^{i} \cdot k^{j}}, \\
& \mathcal{A}_{2}^{\mathrm{PS}}=(2 \pi)^{10} \delta^{(10)}(k) \kappa^{4} e^{2 \lambda} \frac{\sqrt{2} K \bar{K}}{2^{10} \alpha^{\prime 5}}\left(\frac{\alpha^{\prime}}{2}\right)^{10} \int_{\mathcal{M}_{2}} \frac{d^{2} \Omega_{I J}}{\left(\operatorname{det} \operatorname{Im} \Omega_{I J}\right)^{5}} \int_{\Sigma_{4}}\left|\mathcal{Y}_{s}\right|^{2} \prod_{i<j} F_{2}\left(z_{i}, z_{j}\right)^{\alpha k^{i} \cdot k^{j}}(1.2)
\end{aligned}
$$

which explicitly shows that with the pure spinor formalism those coefficients follow directly from a first principles computation. But we find disagreement with the RNS results reported by [33], namely

$$
\mathcal{A}_{1}^{\mathrm{PS}}=\frac{1}{2^{2}} \mathcal{A}_{1}^{\mathrm{RNS}}, \quad \mathcal{A}_{2}^{\mathrm{PS}}=\frac{1}{2^{4}} \mathcal{A}_{2}^{\mathrm{RNS}} .
$$

The mismatches seen in (1.3) will deserve some consideration. On one hand, the previous PS computation of the one-loop coefficient in [28] by one of the authors claimed agreement with the RNS result of [33]. But as will be pointed out in section 4, [28] made a mistake in the evaluation of the b-ghost integral which explains the difference with the computation of this paper. On the other (RNS) hand, we argue in section 4 that [33] forgot the two factors of $1 / 2$ from the GSO projection in the left- and right-moving sectors in their measure. This 
observation will also explain the $1 / 2^{4}$ mismatch at two-loops of section 5 , as [33] fixed the two-loop coefficient using a factorization constraint which depends quadratically on the one-loop coefficient. ${ }^{3}$

In the appendix A we present the detailed covariant computation of the two-loop kinematic factor needed in section 5. This appendix can be regarded as a fully $\mathrm{SO}(10)$ covariant proof of the 2-loop equivalence ${ }^{4}$ between the non-minimal and minimal pure spinor formalisms, and is analogous to the covariant proof of [40] for the 1-loop case. The appendix B is devoted to proving a formula mentioned en passant in [17] which is used to rewrite the two-loop amplitude in terms of integrals in the period matrix instead of in the Teichmüller parameters.

\section{The conventions}

The non-minimal pure spinor formalism action for the left-moving sector reads [13]

$$
S=\frac{1}{2 \pi \alpha^{\prime}} \int_{\Sigma_{g}} d^{2} z\left(\partial X^{m} \bar{\partial} X_{m}+\alpha^{\prime} p_{\alpha} \bar{\partial} \theta^{\alpha}-\alpha^{\prime} \omega_{\alpha} \bar{\partial} \lambda^{\alpha}-\alpha^{\prime} \bar{w}^{\alpha} \overline{\partial \lambda_{\alpha}}+\alpha^{\prime} s^{\alpha} \bar{\partial} r_{\alpha}\right)
$$

with the constraints $\left(\lambda \gamma^{m} \lambda\right)=\left(\bar{\lambda} \gamma^{m} \bar{\lambda}\right)=\left(\bar{\lambda} \gamma^{m} r\right)=0$. The space-time dimensions are the following [28]

$$
\left[\alpha^{\prime}\right]=2,\left[X^{m}\right]=1,\left[\theta^{\alpha}\right]=\left[\lambda^{\alpha}\right]=\left[\bar{\omega}^{\alpha}\right]=\left[s^{\alpha}\right]=1 / 2,\left[p_{\alpha}\right]=\left[\omega_{\alpha}\right]=\left[\bar{\lambda}_{\alpha}\right]=\left[r_{\alpha}\right]=-1 / 2 .
$$

The OPE's for the matter variables following from (2.1) can be computed to be

$$
X^{m}(z) X_{n}(w) \sim-\frac{\alpha^{\prime}}{2} \delta_{n}^{m} \ln |z-w|^{2}, \quad p_{\alpha}(z) \theta^{\beta}(w) \sim \frac{\delta_{a}^{\beta}}{z-w} .
$$

The Green-Schwarz constraint $d_{\alpha}(z)$ and the supersymmetric momentum $\Pi^{m}(z)$ are

$$
d_{\alpha}=p_{\alpha}-\frac{1}{\alpha^{\prime}}\left(\gamma^{m} \theta\right)_{\alpha} \partial X_{m}-\frac{1}{4 \alpha^{\prime}}\left(\gamma^{m} \theta\right)_{\alpha}\left(\theta \gamma_{m} \partial \theta\right), \quad \Pi^{m}=\partial X^{m}+\frac{1}{2}\left(\theta \gamma^{m} \partial \theta\right)
$$

which satisfy the following OPE's

$$
\begin{aligned}
d_{\alpha}(z) d_{\beta}(w) & \sim-\frac{2}{\alpha^{\prime}} \frac{\gamma_{\alpha \beta}^{m} \Pi_{m}}{z-w}, & d_{\alpha}(z) \Pi^{m}(w) & \sim \frac{\gamma_{\alpha \beta}^{m} \partial \theta^{\beta}}{z-w}, \\
d_{\alpha}(z) f(\theta(w), x(w)) & \sim \frac{D_{\alpha} f(\theta(w), x(w))}{z-w}, & \Pi^{m}(z) f(\theta(w), x(w)) & \sim-\frac{\alpha^{\prime}}{2} \frac{k^{m} f(\theta(w), x(w))}{z-w}
\end{aligned}
$$

\footnotetext{
${ }^{3}$ For a compact Riemann surface $S$ of genus $g$ the correct factor is $1 / 2^{2 g}$, which is the number of spin structures over $S$ and is in agreement with factorization.

${ }^{4}$ As will be mentioned in appendix A, there is a loophole in the 2-loop equivalence proof of [20]. Some terms in the non-minimal pure spinor kinematic factor were argued to vanish using a U(5) decomposition but, as will be shown explicitly using the identities of [23], are in fact proportional to the kinematic factor of the minimal pure spinor formalism. As this loophole only affects the proportionality constant, it does not alter the conclusions of [20] but had to be taken into account here.
} 
where $D_{\alpha}=\frac{\partial}{\partial \theta^{\alpha}}+\frac{1}{2}\left(\gamma^{m} \theta\right)_{\alpha} \partial_{m}$ is supersymmetric derivative. The composite b-ghost is given by [13] (see also [41])

$$
\begin{aligned}
b= & s^{\alpha} \partial \bar{\lambda}_{\alpha}+\frac{1}{4(\lambda \bar{\lambda})}\left(2 \Pi^{m}\left(\bar{\lambda} \gamma_{m} d\right)-N_{m n}\left(\bar{\lambda} \gamma^{m n} \partial \theta\right)-J_{\lambda}(\bar{\lambda} \partial \theta)-\left(\bar{\lambda} \partial^{2} \theta\right)\right) \\
& +\frac{\left(\bar{\lambda} \gamma^{m n p} r\right)}{192(\lambda \bar{\lambda})^{2}}\left[\frac{\alpha^{\prime}}{2}\left(d \gamma_{m n p} d\right)+24 N_{m n} \Pi_{p}\right] \\
& -\frac{\alpha^{\prime}}{2} \frac{\left(r \gamma_{m n p} r\right)}{16(\lambda \bar{\lambda})^{3}}\left[\left(\bar{\lambda} \gamma^{m} d\right) N^{n p}-\frac{\left(\bar{\lambda} \gamma^{p q r} r\right) N^{m n} N_{q r}}{8(\lambda \bar{\lambda})}\right]
\end{aligned}
$$

and satisfies [13]

$$
\{Q, b(z)\}=T(z)
$$

where the BRST-charge $Q$ and the energy-momentum tensor $T(z)$ are

$$
Q=\oint\left(\lambda^{\alpha} d_{\alpha}+\bar{w}^{\alpha} r_{\alpha}\right), \quad T(z)=-\frac{1}{\alpha^{\prime}} \partial X^{m} \partial X_{m}-p_{\alpha} \partial \theta^{\alpha}+\omega_{\alpha} \partial \lambda^{\alpha}+\bar{w}^{\alpha} \partial \bar{\lambda}_{\alpha}-s^{\alpha} \partial r_{\alpha} .
$$

From (2.2) it follows that $[Q]=[b]=[T]=0$.

Scattering amplitudes in the non-minimal pure spinor formalism use vertex operators in unintegrated and integrated forms, which for the massless states are given respectively by

$$
V(z)=\lambda^{\alpha} A_{\alpha}, \quad U(z)=\partial \theta^{\alpha} A_{\alpha}+A_{m} \Pi^{m}+\frac{\alpha^{\prime}}{2} d_{\alpha} W^{\alpha}+\frac{\alpha^{\prime}}{4} N_{m n} \mathcal{F}^{m n}
$$

where $A_{\alpha}(X, \theta), A^{m}(X, \theta), W^{\alpha}(X, \theta), \mathcal{F}^{m n}$ are the standard 10-dimensional $\mathcal{N}=1 \mathrm{SYM}$ superfields $[42,43]$. They have the following $\theta$-expansion $[19,44-46]$

$$
\begin{aligned}
A_{\alpha}(x, \theta) & =\frac{1}{2} a_{m}\left(\gamma^{m} \theta\right)_{\alpha}-\frac{1}{3}\left(\xi \gamma_{m} \theta\right)\left(\gamma^{m} \theta\right)_{\alpha}-\frac{1}{32} F_{m n}\left(\gamma_{p} \theta\right)_{\alpha}\left(\theta \gamma^{m n p} \theta\right)+\cdots \\
A_{m}(x, \theta) & =a_{m}-\left(\xi \gamma_{m} \theta\right)-\frac{1}{8}\left(\theta \gamma_{m} \gamma^{p q} \theta\right) F_{p q}+\frac{1}{12}\left(\theta \gamma_{m} \gamma^{p q} \theta\right)\left(\partial_{p} \xi \gamma_{q} \theta\right)+\cdots \\
W^{\alpha}(x, \theta) & =\xi^{\alpha}-\frac{1}{4}\left(\gamma^{m n} \theta\right)^{\alpha} F_{m n}+\frac{1}{4}\left(\gamma^{m n} \theta\right)^{\alpha}\left(\partial_{m} \xi \gamma_{n} \theta\right)+\frac{1}{48}\left(\gamma^{m n} \theta\right)^{\alpha}\left(\theta \gamma_{n} \gamma^{p q} \theta\right) \partial_{m} F_{p q}+\cdots \\
\mathcal{F}_{m n}(x, \theta) & =F_{m n}-2\left(\partial_{[m} \xi \gamma_{n]} \theta\right)+\frac{1}{4}\left(\theta \gamma_{[m} \gamma^{p q} \theta\right) \partial_{n]} F_{p q}+\ldots,
\end{aligned}
$$

where $a_{m}(x)=e_{m} \mathrm{e}^{i k \cdot x}, \xi^{\alpha}(x)=\left(2 / \alpha^{\prime}\right)^{1 / 2} \chi^{\alpha} \mathrm{e}^{i k \cdot x}$ and $F_{m n}=2 \partial_{[m} a_{n]}$ with $\left[e_{m}\right]=0$ and $\left[\chi^{\alpha}\right]=1 / 2$. The space-time dimensions of the superfields and the vertex operators are

$$
\left[A_{\alpha}\right]=1 / 2, \quad\left[A_{m}\right]=0, \quad\left[W^{\alpha}\right]=-1 / 2, \quad\left[\mathcal{F}_{m n}\right]=-1, \quad[V(z)]=[U(z)]=1 .
$$

Vertex operators for the closed string are $V(z, \bar{z})=\tilde{\kappa} V(z) \otimes \tilde{V}(\bar{z})$ and $U(z, \bar{z})=\tilde{\kappa} U(z) \otimes$ $\tilde{U}(\bar{z})$ with the understanding that only the left-moving modes carry the $\mathrm{e}^{i k \cdot x}$ factor. $\tilde{\kappa}$ is the overall vertex operator normalization which will be fixed below to $\tilde{\kappa}=\kappa$, where $\kappa$ is the normalization convention used in [33]. Therefore as in [33], its precise value in terms of $\alpha^{\prime}$ and the string coupling constant [47, 48] will not be needed here.

Finally, the string coupling constant appearing in scattering amplitude computations in the pure spinor formalism is $\mathrm{e}^{(2 g-2) \mu}$. As discussed below, by choosing a convenient 
normalization for the pure spinor tree-level measures its equality with the RNS convention of $[33] \mathrm{e}^{(2 g-2) \mu}=\mathrm{e}^{(2 g-2) \lambda}$ will follow.

The construction of the zero-mode measures for the non-minimal pure spinor variables was given in [13] and their precise normalizations were found in [28]. It is however convenient to use slightly different conventions for the measures of [28] to make their genus- $g$ dependence (and generality) explicit, facilitating computations in different genera. The space-time dimensionless genus- $g$ zero-mode measures are given by

$$
\begin{aligned}
{[d \lambda] T_{\alpha_{1} \alpha_{2} \alpha_{3} \alpha_{4} \alpha_{5}} } & =c_{\lambda} \epsilon_{\alpha_{1} \ldots \alpha_{5} \rho_{1} \ldots \rho_{11}} d \lambda^{\rho_{1}} \ldots d \lambda^{\rho_{11}} \\
{[d \bar{\lambda}] \bar{T}^{\alpha_{1} \alpha_{2} \alpha_{3} \alpha_{4} \alpha_{5}} } & =c_{\bar{\lambda}} \epsilon^{\alpha_{1} \ldots \alpha_{5} \rho_{1} \ldots \rho_{11}} d \bar{\lambda}_{\rho_{1}} \ldots d \bar{\lambda}_{\rho_{11}} \\
{[d \omega] } & =c_{\omega} T_{\alpha_{1} \alpha_{2} \alpha_{3} \alpha_{4} \alpha_{5}} \epsilon^{\alpha_{1} \ldots \alpha_{5} \rho_{1} \ldots \rho_{11}} d \omega_{\rho_{1}} \ldots d \omega_{\rho_{11}} \\
{[d \bar{w}] T_{\alpha_{1} \alpha_{2} \alpha_{3} \alpha_{4} \alpha_{5}} } & =c_{\bar{w}} \epsilon_{\alpha_{1} \ldots \alpha_{5} \rho_{1} \ldots \rho_{11}} d \bar{w}^{\rho_{1}} \ldots d \bar{w}^{\rho_{11}} \\
{[d r] } & =c_{r} \bar{T}^{\alpha_{1} \alpha_{2} \alpha_{3} \alpha_{4} \alpha_{5}} \epsilon_{\alpha_{1} \ldots \alpha_{5} \delta_{1} \ldots \delta_{11}} \partial_{r}^{\delta_{1}} \ldots \partial_{r}^{\delta_{11}} \\
{\left[d s^{I}\right] } & =c_{s} T_{\alpha_{1} \alpha_{2} \alpha_{3} \alpha_{4} \alpha_{5}} \epsilon^{\alpha_{1} \ldots \alpha_{5} \rho_{1} \ldots \rho_{11}} \partial_{\rho_{1}}^{s^{I}} \ldots \partial_{\rho_{11}}^{s^{I}} \\
{[d \theta] } & =c_{\theta} d^{16} \theta
\end{aligned}
$$

with the following normalizations

$$
\begin{aligned}
c_{\lambda} & =\left(\frac{\alpha^{\prime}}{2}\right)^{-2} \frac{1}{11 !}\left(\frac{A_{g}}{4 \pi^{2}}\right)^{11 / 2} & c_{\omega} & =\left(\frac{\alpha^{\prime}}{2}\right)^{2} \frac{\left(4 \pi^{2}\right)^{-11 / 2}}{11 ! 5 ! Z_{g}^{11 / g}} \\
c_{\bar{\lambda}} & =\left(\frac{\alpha^{\prime}}{2}\right)^{2} \frac{2^{6}}{11 !}\left(\frac{A_{g}}{4 \pi^{2}}\right)^{11 / 2} & c_{\bar{w}} & =\left(\frac{\alpha^{\prime}}{2}\right)^{-2} \frac{\left(4 \pi^{2}\right)^{-11 / 2}(\lambda \bar{\lambda})^{3}}{11 ! Z_{g}^{11 / g}} \\
c_{r} & =\left(\frac{\alpha^{\prime}}{2}\right)^{-2} \frac{R}{11 ! 5 !}\left(\frac{2 \pi}{A_{g}}\right)^{11 / 2} & c_{s} & =\left(\frac{\alpha^{\prime}}{2}\right)^{2} \frac{(2 \pi)^{11 / 2} R^{-1}}{2^{6} 11 ! 5 !(\lambda \bar{\lambda})^{3}} Z_{g}^{11 / g} \\
c_{\theta} & =\left(\frac{\alpha^{\prime}}{2}\right)^{4}\left(\frac{2 \pi}{A_{g}}\right)^{16 / 2} & c_{d} & =\left(\frac{\alpha^{\prime}}{2}\right)^{-4}(2 \pi)^{16 / 2} Z_{g}^{16 / g}
\end{aligned}
$$

where $R$ is arbitrary and parametrizes the freedom in choosing the normalization of the tree-level amplitude and $A_{g}$ is the area of the Riemann surface. As will be shown in section 3 , using the value

$$
R^{2}=\frac{\sqrt{2}}{2^{16} \pi}
$$

fixes the tree-level normalization to be the same as in the RNS computations of [33]. The tensors $T_{\alpha_{1} \ldots \alpha_{5}}, \bar{T}^{\alpha_{1} \ldots \alpha_{5}}$ are defined as

$$
\begin{aligned}
T_{\alpha_{1} \alpha_{2} \alpha_{3} \alpha_{4} \alpha_{5}} & =\left(\lambda \gamma^{m}\right)_{\alpha_{1}}\left(\lambda \gamma^{n}\right)_{\alpha_{2}}\left(\lambda \gamma^{p}\right)_{\alpha_{3}}\left(\gamma_{m n p}\right)_{\alpha_{4} \alpha_{5}} \\
\bar{T}^{\alpha_{1} \alpha_{2} \alpha_{3} \alpha_{4} \alpha_{5}} & =\left(\bar{\lambda} \gamma^{m}\right)^{\alpha_{1}}\left(\bar{\lambda} \gamma^{n}\right)^{\alpha_{2}}\left(\bar{\lambda} \gamma^{p}\right)^{\alpha_{3}}\left(\gamma_{m n p}\right)^{\alpha_{4} \alpha_{5}}
\end{aligned}
$$

and satisfy

$$
T_{\alpha_{1} \alpha_{2} \alpha_{3} \alpha_{4} \alpha_{5}} \bar{T}^{\alpha_{1} \alpha_{2} \alpha_{3} \alpha_{4} \alpha_{5}}=5 ! 2^{6}(\lambda \bar{\lambda})^{3} .
$$

The appearance of the area $A_{g}$ and of the factor $Z_{g}$ will be explained in the next subsection. They are

$$
A_{g}=\int d^{2} z \sqrt{g}, \quad Z_{g}=\frac{1}{\sqrt{\operatorname{det}\left(2 \operatorname{Im}\left(\Omega_{I J}\right)\right)}}, \quad g \geq 1
$$


where $\Omega_{I J}$ is the period matrix of the Riemann surface. It is well-known that for $g=1$ the period matrix is given by the Teichmüller parameter $\tau$.

To avoid cluttering in the formulæ we define the genus $g$ bracket \langle\rangle$_{(n, g)}$ as

$$
\langle M(\lambda, \bar{\lambda}, \theta)\rangle_{(n, g)} \equiv \int[d \theta][d r][d \lambda][d \bar{\lambda}] \frac{e^{-(\lambda \bar{\lambda})-(r \theta)}}{(\lambda \bar{\lambda})^{3-n}} M(\lambda, \bar{\lambda}, \theta, r)
$$

for an arbitrary pure spinor superfield $M(\lambda, \bar{\lambda}, \theta, r)$. With the above conventions the integral over the zero modes of pure spinor space becomes [28]

$$
\int[d \lambda][d \bar{\lambda}](\lambda \bar{\lambda})^{n} \mathrm{e}^{-(\lambda \bar{\lambda})}=\frac{(7+n) !}{7 ! 60}\left(\frac{2 \pi}{A_{g}}\right)^{11}, \quad n \geq 0
$$

which together with (2.23) imply that

$$
N_{(n, g)} \equiv\left\langle\lambda^{3} \theta^{5}\right\rangle_{(n, g)}=2^{7} R\left(\frac{2 \pi}{A_{g}}\right)^{5 / 2}\left(\frac{\alpha^{\prime}}{2}\right)^{2} \frac{(7+n) !}{7 !}, \quad n \geq 0
$$

where we used the abbreviated notation $\left(\lambda^{3} \theta^{5}\right)=\left(\lambda \gamma^{r} \theta\right)\left(\lambda \gamma^{s} \theta\right)\left(\lambda \gamma^{t} \theta\right)\left(\theta \gamma_{r s t} \theta\right)$. Due to the identities of [23] the following trick from [28] is required for the tree-level, one- and two-loop amplitudes

$$
\left\langle\left(\lambda A^{1}\right)\left(\lambda \gamma^{m} W^{2}\right)\left(\lambda \gamma^{n} W^{3}\right) \mathcal{F}_{m n}^{4}\right\rangle_{(n, g)}=-\frac{K}{2^{9} 3^{2} 5}\left\langle\left(\lambda^{3} \theta^{5}\right)\right\rangle_{(n, g)}
$$

where $K$ denotes the kinematic factor of [33], which will be written down below.

It is convenient to consider the genus-g expectation value of the exponentials at the same time as the integration over the non-zero modes of the pure spinor variables, as the latter is equal to $(\operatorname{det} \partial \bar{\partial})^{5}$ [28]. When both expressions are computed the determinant factors cancels out and one can use the following expression

$$
\left\langle\prod_{i=1}^{4} e^{i k \cdot x}\right\rangle_{g}=(2 \pi)^{10} \delta^{(10)}(k) \frac{A_{g}^{5}}{\left(2 \pi^{2} \alpha^{\prime}\right)^{5}} \prod_{i<j} F_{g}\left(z_{i}, z_{j}\right)^{\alpha k^{i} \cdot k^{j}}
$$

for their combined result. Therefore by using (2.29) the integration over non-zero modes of the pure spinor variables is already taken care of. For the sphere one has $F_{0}\left(z_{i}, z_{j}\right)=\left|z_{i j}\right|$ whereas for genus $g \geq 1$ it can be written in terms of the prime form as [1]

$$
F_{g}\left(z_{i}, z_{j}\right)^{\alpha k^{i} \cdot k^{j}}=\left|E\left(z_{i}, z_{j}\right)\right|^{\alpha k^{i} \cdot k^{j}} \exp \left(-2 \pi(\operatorname{Im} \Omega)_{I J}^{-1}\left(\operatorname{Im} \int_{z_{i}}^{z_{j}} w_{I}\right)\left(\operatorname{Im} \int_{z_{i}}^{z_{j}} w_{J}\right)\right),
$$

where $w_{I}(z)(I=1, \ldots, g)$ are the holomorphic 1-forms over $\Sigma_{g}$.

From (2.27) and (2.29) it follows that in amplitudes of closed string states the factors of $A_{g}$ cancel in the always-present product of,

$$
\left|N_{(n, g)}\right|^{2}\left\langle\prod_{i=1}^{N} e^{i k \cdot x}\right\rangle_{g}=(2 \pi)^{10} \delta^{(10)}(k) \frac{\sqrt{2}}{2^{2} \pi^{6} \alpha^{\prime 5}}\left(\frac{\alpha^{\prime}}{2}\right)^{4}\left(\frac{(7+n) !}{7 !}\right)^{2} \prod_{i<j} F_{g}\left(z_{i}, z_{j}\right)^{\alpha k^{i} \cdot k^{j}} .
$$


The independence of the closed string amplitude with respect to the area of the surface follows from the fact that the number of bosonic and fermionic conformal weight-zero variables is the same.

The topological prescription [13] for computing the 4-point amplitudes at tree-level, one- and two-loops ${ }^{5}$ is

$$
\begin{aligned}
& \mathcal{A}_{0}=\tilde{\kappa}^{4} e^{-2 \mu} \int d^{2} z_{4}\left\langle\left|\mathcal{N} V^{1}(0) V^{2}(1) V^{3}(\infty) U^{4}\left(z_{4}\right)\right|^{2}\right\rangle \\
& \mathcal{A}_{1}=\frac{1}{2} \tilde{\kappa}^{4} \int_{\mathcal{M}_{1}} d^{2} \tau_{1} \prod_{i=2}^{4} \int d^{2} z_{i}\left\langle\left|\mathcal{N}\left(b, \mu_{1}\right) V^{1}(0) U^{i}\left(z_{i}\right)\right|^{2}\right\rangle \\
& \mathcal{A}_{2}=\frac{1}{2} \tilde{\kappa}^{4} e^{2 \mu} \int_{\mathcal{M}_{2}} \prod_{I=1}^{3} d^{2} \tau_{I} \prod_{i=1}^{4} \int d^{2} z_{i}\left\langle\left|\mathcal{N}\left(b, \mu_{I}\right) U^{i}\left(z_{i}\right)\right|^{2}\right\rangle
\end{aligned}
$$

where $\mathcal{M}_{1}\left(\mathcal{M}_{2}\right)$ is the fundamental domain of the Riemann surface of genus 1 (genus 2) and $\mathcal{N}$ is the regulator [13]

$$
\mathcal{N}=\sum_{I=1}^{g} \mathrm{e}^{-(\lambda \bar{\lambda})-\left(w^{I} \bar{w}^{I}\right)-(r \theta)+\left(s^{I} d^{I}\right)}
$$

\langle\rangle denotes the integrations over the zero-modes

$$
\langle\rangle \rightarrow \prod_{I=1}^{g} \int[d \theta]\left[d d^{I}\right][d r]\left[d s^{I}\right]\left[d \bar{w}^{I}\right]\left[d w^{I}\right][d \lambda][d \bar{\lambda}]
$$

and the b-ghost insertion is $[50,51]$

$$
\left(b, \mu_{j}\right)=\frac{1}{2 \pi} \int d^{2} y_{j} b_{z z} \mu_{j \bar{z}}^{z}, \quad j=1, \ldots, 3 g-3 .
$$

where the normalization $1 / 2 \pi$ comes from bosonic string theory [50] because the topological prescription is based on it. With the above conventions, the space-time dimension of the genus- $g$ four-point amplitudes is given by $\left[\mathcal{A}_{g}\right]=8$. In the following sections we don't keep track of the overall sign of the amplitudes.

Following [33] we use $d^{2} \tau=d \tau \wedge d \bar{\tau}, d^{2} z=d z \wedge d \bar{z}$ (in particular $\int_{\Sigma_{1}} d^{2} z=2 \tau_{2}$ ). Furthermore $\mathcal{Y}_{s}$ has space-time dimension -2 and is given by

$$
\mathcal{Y}_{s}=-s \Delta(1,4) \Delta(2,3)+t \Delta(1,2) \Delta(3,4),
$$

where $\Delta(i, j) \equiv w_{1}\left(z_{i}\right) w_{2}\left(z_{j}\right)-w_{1}\left(z_{j}\right) w_{2}\left(z_{i}\right)$ and $w_{I}(z)$ is the basis of holomorphic 1-forms discussed below and $s=-2\left(k^{1} \cdot k^{2}\right), t=-2\left(k^{2} \cdot k^{3}\right), u=-2\left(k^{1} \cdot k^{3}\right)$ are the Mandelstam variables satisfying $s+t+u=0$. Finally, the omnipresent supersymmetric kinematic factor $K$ can be conveniently represented by the pure spinor superspace expression

\footnotetext{
${ }^{5}$ The $\frac{1}{2}$ factor appearing in the two-loop amplitude was argued for in [49]. Every Riemann surface of genus 2 can be written like a hyperelliptic curve $y^{2}=h(z)$ where $h(z)$ is a polynomial of degree 6 and $y$ is the coordinate over $C P^{1}$. This curve has the $Z_{2}$ symmetry $y \rightarrow-y$, so the $1 / 2$ factor is needed. We would like to thank Cumrun Vafa for this explanation.
} 
$K=23040\left\langle\left(\lambda A^{1}\right)\left(\lambda \gamma^{m} W^{2}\right)\left(\lambda \gamma^{n} W^{3}\right) \mathcal{F}_{m n}^{4}\right\rangle$, where the brackets here are defined such that $\left\langle\left(\lambda^{3} \theta^{5}\right)\right\rangle=1$ [23]. While the computations of [33] did not involve the whole supermultiplet, this representation of $K$ is convenient because its bosonic component expansion has the same normalization of the kinematic factor $K$ of [33],

$$
K=\left(e^{1} \cdot e^{2}\right)\left[2 t u\left(e^{3} \cdot e^{4}\right)-4 t\left(k^{1} \cdot e^{3}\right)\left(k^{2} \cdot e^{4}\right)\right]+\text { perm }+ \text { fermions }
$$

where the fermionic terms can be looked up in [23].

\subsection{The normalization of zero-modes}

Since the dimension of the zero Čech cohomology group $H^{0}\left(\Sigma_{g}, \Omega^{1}\right)$, where $\Omega^{1}\left(\Sigma_{g}\right)$ is the sheaf of holomorphic 1-forms over $\Sigma_{g}$, is equal to the genus $g$ of the Riemann surface we expand a generic conformal weight $(1,0)$ field as [13]

$$
\phi(z)=\hat{\phi}(z)+\sum_{i=1}^{g} w_{i}(z) \phi^{i}
$$

where $\phi^{i}$ are the zero modes and $\left\{w_{i}(z) d z\right\}$ is a basis of the $H^{0}\left(\Sigma_{g}, \Omega^{1}\right)$ group such that

$$
\begin{aligned}
\int_{a_{i}} w_{j}(z) d z & =\delta_{i j}, \quad \int_{b_{i}} w_{j}(z) d z=\Omega_{i j} \quad i, j=1,2, \ldots, g \\
\left(w_{i}, w_{j}\right) & \equiv \int_{\Sigma_{g}} w_{i} \bar{w}_{j} d z \wedge d \bar{z}=2 \operatorname{Im} \Omega_{\mathrm{ij}}
\end{aligned}
$$

where $a_{i}$ and $b_{j}$ are the generators of the $H^{1}\left(\Sigma_{g}, Z\right)=Z^{2 g}$ homology group and $\Omega_{i j}$ is the period matrix [52]. If we expand $\phi$ over another basis $\left\{\alpha_{j}\right\}$ related by $w_{i}=B_{i}^{j} \alpha_{j}$ then [53],

$$
\operatorname{det}\left(\int_{\Sigma_{g}} w_{i} \bar{w}_{j} d z \wedge d \bar{z}\right)=\operatorname{det}|B|^{2} \operatorname{det}\left(\int_{\Sigma_{g}} \alpha_{i} \bar{\alpha}_{j} d z \wedge d \bar{z}\right)
$$

so that for

$$
|\operatorname{det} B|=\sqrt{\operatorname{det}\left(2 \operatorname{Im} \Omega_{i j}\right)}=Z_{g}^{-1}
$$

the basis $\left\{\alpha_{j}\right\}$ is orthonormal, $\left(\alpha_{i}, \alpha_{j}\right)=\delta_{i j}$. Expanding the fields over the new basis as $\phi=\sum_{j=1}^{g} \phi^{\prime j} \alpha_{j}$ one can show that the measure satisfies

$$
d \phi^{1} \cdots d \phi^{\prime g}=\operatorname{det}(\mathrm{B})^{\epsilon} d \phi^{1} \cdots d \phi^{g},
$$

where $\epsilon=+1(-1)$ for bosonic (fermionic) fields. In the non-minimal formalism the integration measures for conformal weight-one fields is defined in terms of the $\phi^{\prime}$ components, but it is more convenient to use the $\left\{w_{I}\right\}$ basis in explicit computations. To account for this we absorb the Jacobian (2.42) equally into each of the $\left[d \phi^{I}\right]$ measures as $\left(\operatorname{det}(\mathrm{B})^{\epsilon / g} d \phi^{1}\right) \cdots\left(\operatorname{det}(\mathrm{B})^{\epsilon / g} d \phi^{g}\right)$, which explains the factors of $Z_{g}$ in $(2.16)-(2.19)$.

Similarly, the appearance of $A_{g}$ in the measures of the conformal weight-zero variables $\left[\lambda^{\alpha}, \bar{\lambda}_{\alpha}, r_{\alpha}, \theta^{\alpha}\right]$ follows from the expansion in a complete set of eigenfunctions for the Laplacian of the worldsheet [54]

$$
\lambda^{\alpha}(z)=\lambda_{0}^{\alpha} \Lambda_{0}+\sum_{j} \lambda_{j}^{\alpha} \Lambda_{j}(z, \bar{z})
$$

and $\Lambda_{0}=1$ is the generator of the cohomology group $H^{0}\left(\Sigma_{g}, \mathcal{O}\right)=\mathbf{C}$, where $\mathcal{O}$ is the sheaf of holomorphic functions over $\Sigma_{g}$. Because the norm of $\Lambda_{0}$ is $\left\|\Lambda_{0}\right\|^{2}=A_{g}$ the measures of the scalars must have the Jacobian $A_{g}^{\epsilon / 2}$ (where $\epsilon=+1(-1)$ for bosonic (fermionic) fields), explaining the factors of $A_{g}$ in (2.16)-(2.19). 


\subsection{On the normalization of the holomorphic 1-forms}

The result of scattering amplitudes in the pure spinor formalism does not depend on the normalization of the holomorphic 1-forms $w_{I}(z)$. To see this one notes that in closed string amplitudes $^{6}$ at genus $g$ the difference between the number of independent fermionic and bosonic conformal weight-one left-moving variables is always $16 g+11 g-11 g-11 g=$ $5 g$, corresponding to $d_{\alpha}^{I}, s^{\alpha I}, w_{\alpha}^{I}$ and $\bar{w}^{\alpha I}$. As $Z_{g}$ appear in the conformal weight-one measures as $Z_{g}^{1 / g}$, their total contribution to closed string amplitudes is always $\left|Z_{g}^{5}\right|^{2}=Z_{g}^{10}$. Furthermore, when saturating the $11 g s^{\alpha I}$ zero modes the regulator factor $\mathcal{N}$ provides $11 g$ $d_{\alpha}^{I}$ zero-modes as well - because they appear in the combination $\left(s^{I} d^{I}\right)$ in $\mathcal{N}$ and there is nowhere else to get $s^{I \alpha}$ zero-modes from. So to complete the saturation of $d_{\alpha}^{I}$ the bghosts and external vertices will always provide $5 g$ factors of $\left|d_{\alpha}^{I} w_{I}(z)\right|^{2}$, which scales as $x^{10 g}$ under $w_{I}(z) \rightarrow x w_{I}(z)$. To finish the proof it suffices to note from (2.41) and (2.42) that $Z_{g}$ scales as $Z_{g} \rightarrow x^{-g} Z_{g}$ and therefore $\left|Z_{g}^{5}\right|^{2}$ offsets the scaling of the $\left|w_{I}^{5 g}\right|^{2}$ factors from the b-ghosts and external vertices.

\section{Tree-level}

The massless four-point amplitude at tree-level is given by (2.32),

$$
\mathcal{A}_{0}=\tilde{\kappa}^{4} e^{-2 \mu} \int d^{2} z_{4}\left\langle\left|\mathcal{N} V^{1}(0) V^{2}(1) V^{3}(\infty) U^{4}\left(z_{4}\right)\right|^{2}\right\rangle .
$$

The amplitude (3.1) was computed in components by [19] and later expressed in pure spinor superspace up to an overall normalization in [23], where it was used that $\left\langle\prod_{i=1}^{4} e^{i k^{i} x\left(z_{i}, \bar{z}_{i}\right)}\right\rangle=$ $\left|z_{4}\right|^{-\frac{1}{2} \alpha^{\prime} t}\left|1-z_{4}\right|^{-\frac{1}{2} \alpha^{\prime} u}$. The normalization of the tree-level amplitude of [23] can be determined a posteriori by using the precise value for the expectation value of the exponentials,

$$
\left\langle\prod_{i=1}^{4} e^{i k^{i} x\left(z_{i}, \bar{z}_{i}\right)}\right\rangle_{0}=(2 \pi)^{10} \delta^{(10)}(k)\left(\frac{A_{0}}{2 \pi^{2} \alpha^{\prime}}\right)^{5}\left|z_{4}\right|^{-\frac{1}{2} \alpha^{\prime} t}\left|1-z_{4}\right|^{-\frac{1}{2} \alpha^{\prime} u},
$$

where $A_{0}=4 \pi$ is the area of the sphere. Doing that in the computations of [23] we obtain,

$$
\mathcal{A}_{0}=(2 \pi)^{10} \delta^{(10)}(k) \tilde{\kappa}^{4} e^{-2 \mu}\left(\frac{4 \pi}{2 \pi^{2} \alpha^{\prime}}\right)^{5}\left(\frac{\alpha^{\prime}}{2}\right)^{4} K_{0} \bar{K}_{0} C(s, t, u),
$$

where

$$
C(s, t, u)=2 \pi \frac{\Gamma\left(-\frac{\alpha^{\prime} s}{4}\right) \Gamma\left(-\frac{\alpha^{\prime} t}{4}\right) \Gamma\left(-\frac{\alpha^{\prime} u}{4}\right)}{\Gamma\left(1+\frac{\alpha^{\prime} s}{4}\right) \Gamma\left(1+\frac{\alpha^{\prime} t}{4}\right) \Gamma\left(1+\frac{\alpha^{\prime} u}{4}\right)}
$$

and the kinematic factor $K_{0}$ is given by the pure spinor superspace expression [23]

$$
K_{0}=\left\langle\left(\lambda A^{1}\right)\left(\lambda \gamma^{m} W^{2}\right)\left(\lambda \gamma^{n} W^{3}\right) \mathcal{F}_{m n}^{4}\right\rangle_{(3,0)}=-\frac{K}{2^{9} 3^{2} 5}\left\langle\left(\lambda^{3} \theta^{5}\right)\right\rangle_{(3,0)}
$$

where the last equality follows from (2.28). Using (2.27) we get

$$
K_{0}=K \frac{N^{(3,0)}}{\left(2^{9} 3^{2} 5\right)}=\frac{R}{\sqrt{2}}\left(\frac{\alpha^{\prime}}{2}\right)^{2} K
$$

\footnotetext{
${ }^{6}$ The analysis can be trivially modified to the open string.
} 
and therefore

$$
\begin{aligned}
\mathcal{A}_{0} & =(2 \pi)^{10} \delta^{(10)}(k) \tilde{\kappa}^{4} e^{-2 \mu} \frac{R^{2}}{2}\left(\frac{2}{\pi \alpha^{\prime}}\right)^{5}\left(\frac{\alpha^{\prime}}{2}\right)^{8} K \bar{K} C(s, t, u) \\
& =(2 \pi)^{10} \delta^{(10)}(k) \tilde{\kappa}^{4} e^{-2 \mu}\left(\frac{\sqrt{2}}{2^{12} \pi^{6} \alpha^{\prime 5}}\right)\left(\frac{\alpha^{\prime}}{2}\right)^{8} K \bar{K} C(s, t, u),
\end{aligned}
$$

where we used that $R^{2}=\frac{\sqrt{2}}{2^{16} \pi}$.

\subsection{The tree-level normalization}

To fix the normalizations at tree-level to match those of [33] we need two conditions [47, 48], therefore we also evaluate the three-point amplitude, which is given by

$$
\mathcal{A}_{\mathrm{t}}=\tilde{\kappa}^{3} e^{-2 \mu}\left\langle|\mathcal{N} V(0) V(1) V(\infty)|^{2}\right\rangle .
$$

Using (2.29), the component expansion found in [40] and the fact that $\left(k^{i} \cdot k^{j}\right)=0$

$$
\mathcal{A}_{\mathrm{t}}=(2 \pi)^{10} \delta^{(10)}(k) \tilde{\kappa}^{3} e^{-2 \mu} \frac{A_{0}^{5}}{\left(2 \pi^{2} \alpha^{\prime}\right)^{5}}\left|K_{t}\right|^{2}
$$

hence,

$$
\mathcal{A}_{\mathrm{t}}=(2 \pi)^{10} \delta^{(10)}(k) \tilde{\kappa}^{3} e^{-2 \mu} \frac{\sqrt{2}}{2^{6} \pi^{6} \alpha^{\prime 5}}\left(\frac{\alpha^{\prime}}{2}\right)^{4} W_{3} \bar{W}_{3}
$$

where we used that

$$
\left|K_{t}\right|^{2}=\left|\left\langle\left(\lambda A^{1}\right)\left(\lambda A^{2}\right)\left(\lambda A^{3}\right)\right\rangle_{(3,0)}\right|^{2}=\frac{\left|N_{(3,0)}\right|^{2}}{2880^{2}} W_{3} \bar{W}_{3}=\frac{\sqrt{2}}{2^{6} \pi}\left(\frac{2 \pi}{A_{0}}\right)^{5}\left(\frac{\alpha^{\prime}}{2}\right)^{4} W_{3} \bar{W}_{3}
$$

and $W_{3}=\left(e^{1} \cdot e^{2}\right)\left(k^{2} \cdot e^{3}\right)+\left(e^{1} \cdot e^{3}\right)\left(k^{1} \cdot e^{2}\right)+\left(e^{2} \cdot e^{3}\right)\left(k^{3} \cdot e^{1}\right)$ is the 3-pt kinematic factor in the RNS computation of [33].

In the normalization conventions of [33] the tree-level tree- and four-point amplitudes were shown to be given by $^{7}$

$$
\begin{aligned}
& \mathcal{A}_{\mathrm{t}}^{\mathrm{RNS}}=(2 \pi)^{10} \delta^{(10)}(k) \kappa^{3} e^{-2 \lambda}\left(\frac{\sqrt{2}}{2^{6} \pi^{6} \alpha^{\prime 5}}\right)\left(\frac{\alpha^{\prime}}{2}\right)^{4} W_{3} \bar{W}_{3}, \\
& \mathcal{A}_{0}^{\mathrm{RNS}}=(2 \pi)^{10} \delta^{(10)}(k) \kappa^{4} e^{-2 \lambda}\left(\frac{\sqrt{2}}{2^{12} \pi^{6} \alpha^{\prime 5}}\right)\left(\frac{\alpha^{\prime}}{2}\right)^{8} K \bar{K} C(s, t, u) .
\end{aligned}
$$

Comparing the RNS results of (3.10) and (3.11) with the corresponding PS amplitudes of (3.9) and (3.7) it follows that

$$
\tilde{\kappa}=\kappa, \quad e^{-2 \mu}=e^{-2 \lambda},
$$

so the PS and RNS tree-level normalization conventions are the same. The numerical value of the parameter $R$ in (2.20) was chosen precisely for this match to happen. After this tree-level matching is done there remains no more freedom to adjust conventions.

\footnotetext{
${ }^{7}$ Note that $\left[\mathcal{A}_{t}\right]=6$ and $\left[\mathcal{A}_{0}\right]=8$, so in $[33]$ the factors of $\left(\alpha^{\prime} / 2\right)$ were forgotten.
} 


\section{One-loop}

The one-loop massless four-point amplitude is given by (2.33),

$$
\mathcal{A}_{1}=\frac{1}{2} \kappa^{4} \int_{\mathcal{M}_{1}} d^{2} \tau_{1} \prod_{i=2}^{4} \int d^{2} z_{i}\left\langle\left|\mathcal{N}\left(b, \mu_{1}\right) V^{1}(0) U^{i}\left(z_{i}\right)\right|^{2}\right\rangle .
$$

The regulator in $(2.35)$ becomes $\mathcal{N}=\mathrm{e}^{-(\lambda \bar{\lambda})-\left(w^{1} \bar{w}^{1}\right)-(r \theta)+\left(s^{1} d^{1}\right)},\langle\rangle$ denotes the integrations over the zero-modes of $\left[\theta^{\alpha}, d_{\alpha}, r_{\alpha}, s^{\alpha}, w_{\alpha}, \bar{w}^{\alpha}, \lambda^{\alpha}, \bar{\lambda}_{\alpha}\right]$ and the b-ghost insertion written in (2.37) reads

$$
\left(b, \mu_{1}\right)=\frac{1}{2 \pi} \int d^{2} z b_{z z} \mu_{\bar{z}}^{z} .
$$

As discussed in [13], there is an unique way to saturate the zero-modes of all variables. The b-ghost must provide two $d_{\alpha}^{1}$ zero-modes with $\frac{1}{2^{63}}\left(\frac{\alpha^{\prime}}{2}\right)\left(\bar{\lambda} \gamma^{m n p} r\right)\left(d^{1} \gamma_{m n p} d^{1}\right) w_{1} w_{1}$, where $w_{1}=1$ is the holomorphic 1 -form in the torus. Therefore the integral (4.2) is easily computed to give

$$
\left(b, \mu_{1}\right)=\frac{1}{2^{7} 3 \pi}\left(\frac{\alpha^{\prime}}{2}\right) \frac{\left(\bar{\lambda} \gamma^{m n p} r\right)\left(d^{1} \gamma_{m n p} d^{1}\right)}{(\lambda \bar{\lambda})^{2}},
$$

because $\int d^{2} z w_{1} w_{1} \mu_{1}=1$. The integrated vertices contribute three $d_{\alpha}^{1}$ zero-modes via $\left(\frac{\alpha^{\prime}}{2}\right)^{3}\left(d^{1} W^{2}\right)\left(d^{1} W^{3}\right)\left(d^{1} W^{4}\right)$, so (4.1) becomes

$$
\mathcal{A}_{1}=\frac{1}{2^{15} 3^{2} \pi^{2}} \kappa^{4}\left(\frac{\alpha^{\prime}}{2}\right)^{8} \int_{\mathcal{M}_{1}} d^{2} \tau \prod_{i=2}^{4} \int d^{2} z_{i}\left|\mathcal{K}_{1}\right|^{2}\left\langle\prod_{i=1}^{4} \mathrm{e}^{i k X\left(z_{i}\right)}\right\rangle_{1},
$$

where the computation of the zero-mode integrations in

$$
\begin{aligned}
\mathcal{K}_{1}= & \int\left[d d^{1}\right]\left[d s^{1}\right]\left[d w^{1}\right]\left[d \bar{w}^{1}\right] \mathrm{e}^{-\left(w^{1} \bar{w}^{1}\right)+\left(s^{1} d^{1}\right)} \times \\
& \times\left\langle\left(\bar{\lambda} \gamma^{m n p} r\right)\left(d^{1} \gamma_{m n p} d^{1}\right)\left(\lambda A^{1}\right)\left(d^{1} W^{2}\right)\left(d^{1} W^{3}\right)\left(d^{1} W^{4}\right)\right\rangle_{(1,1)}
\end{aligned}
$$

is straightforward and goes as follows. Using the measures (2.11) and (2.12) and the results of [28] one gets

$$
\int[d w][d \bar{w}] \mathrm{e}^{-(w \bar{w})}=\frac{(\lambda \bar{\lambda})^{3}}{(2 \pi)^{11} Z_{1}^{22}}
$$

Hence,

$$
\mathcal{K}_{1}=\frac{1}{(2 \pi)^{11} Z_{1}^{22}} \int\left[d d^{1}\right]\left[d s^{1}\right] \mathrm{e}^{\left(s^{1} d^{1}\right)}\left\langle\left(\bar{\lambda} \gamma^{m n p} r\right)\left(d \gamma_{m n p} d\right)\left(\lambda A^{1}\right)\left(d W^{2}\right)\left(d W^{3}\right)\left(d W^{4}\right)\right\rangle_{(4,1)} .
$$

The integration over $[d s]$ using the measure (2.14) leads to

$$
\begin{aligned}
\mathcal{K}_{1}= & \frac{(2 \pi)^{-11 / 2}}{2^{6}(11 ! 5 !) Z_{1}^{11} R}\left(\frac{\alpha^{\prime}}{2}\right)^{2} \int\left[d d^{1}\right] T_{\alpha_{1} \ldots \alpha_{5}} \epsilon^{\alpha_{1} \ldots \alpha_{5} \delta_{1} \ldots \delta_{11}} d_{\delta_{1}} \ldots d_{\delta_{11}} \\
& \times\left\langle\left(\bar{\lambda} \gamma^{m n p} r\right)\left(d^{1} \gamma_{m n p} d^{1}\right)\left(\lambda A^{1}\right)\left(d^{1} W^{2}\right)\left(d^{1} W^{3}\right)\left(d^{1} W^{4}\right)\right\rangle_{(1,1)} .
\end{aligned}
$$

Using the identities

$$
\int d^{16} d d_{\rho_{1}} \ldots d_{\rho_{16}}=\epsilon_{\rho_{1} \ldots \rho_{16}}, \epsilon_{\rho_{1} \ldots \rho_{16}} \epsilon^{\alpha_{1} \ldots \alpha_{5} \rho_{1} \ldots \rho_{11}}=11 ! 5 ! \delta_{\rho_{12} \ldots \rho_{16}}^{\alpha_{1} \ldots \alpha_{5}}(, .8)
$$




$$
\begin{gathered}
\left(\gamma^{a b c}\right)^{\rho_{12} \rho_{13}}\left(\gamma_{m_{1} n_{1} p_{1}}\right)_{\rho_{12} \rho_{13}}=-2^{5} 3 \delta_{m_{1} n_{1} p_{1}}^{a b c} \\
\left(\lambda \gamma^{m_{1}}\right)_{\left[\alpha_{1}\right.}\left(\lambda \gamma^{n_{1}}\right)_{\alpha_{2}}\left(\lambda \gamma^{p_{1}}\right)_{\alpha_{3}}\left(\gamma_{m_{1} n_{1} p_{1}}\right)_{\left.\alpha_{4} \alpha_{5}\right]}=\left(\lambda \gamma^{m_{1}}\right)_{\alpha_{1}}\left(\lambda \gamma^{n_{1}}\right)_{\alpha_{2}}\left(\lambda \gamma^{p_{1}}\right)_{\alpha_{3}}\left(\gamma_{m_{1} n_{1} p_{1}}\right)_{\alpha_{4} \alpha_{5}}
\end{gathered}
$$

the integration over $\left[d d^{1}\right]$ is easily performed and (4.7) becomes

$$
\mathcal{K}_{1}=\frac{3(2 \pi)^{5 / 2} Z_{1}^{5}}{2 R}\left(\frac{\alpha^{\prime}}{2}\right)^{-2}\left\langle\left(\bar{\lambda} \gamma^{m n p} D\right)\left(\lambda A^{1}\right)\left(\lambda \gamma_{m} W^{2}\right)\left(\lambda \gamma_{n} W^{3}\right)\left(\lambda \gamma_{p} W^{4}\right)\right\rangle_{(1,1)}
$$

where we also used that $[20] \int \mathrm{e}^{-(r \theta)} r_{\alpha}(\ldots)=\int D_{\alpha} \mathrm{e}^{-(r \theta)}(\ldots)$. Using the identity [40]

$$
\begin{gathered}
\left\langle\left(\bar{\lambda} \gamma^{m n p} D\right)\left(\lambda A^{1}\right)\left(\lambda \gamma_{m} W^{2}\right)\left(\lambda \gamma_{n} W^{3}\right)\left(\lambda \gamma_{p} W^{4}\right)\right\rangle_{(1,1)}=40\left\langle\left(\lambda A^{1}\right)\left(\lambda \gamma^{m} W^{2}\right)\left(\lambda \gamma^{n} W^{3}\right) \mathcal{F}_{m n}^{4}\right\rangle_{(2,1)} \\
=\frac{K}{2^{6} 3^{2}}\left\langle\left(\lambda^{3} \theta^{5}\right)\right\rangle_{(2,1)}
\end{gathered}
$$

where in the last line we used (2.28), the kinematic factor (4.11) can be written as

$$
\mathcal{K}_{1}=\frac{(2 \pi)^{5 / 2} Z_{1}^{5} K}{3 R 2^{7}}\left(\frac{\alpha^{\prime}}{2}\right)^{-2}\left\langle\left(\lambda^{3} \theta^{5}\right)\right\rangle_{(2,1)}
$$

Using the definition (2.27) one concludes from (4.12) that

$$
\left|\left\langle\mathcal{K}_{1}\right\rangle\right|^{2}=\frac{(2 \pi)^{5} Z_{1}^{10}}{2^{14} 3^{2} R^{2}} K \bar{K}\left|N_{(2,1)}\right|^{2}\left(\frac{\alpha^{\prime}}{2}\right)^{-4} .
$$

The amplitude (4.3) therefore is given by

$$
\mathcal{A}_{1}=\frac{(2 \pi)^{5}}{2^{29} 3^{4} R^{2} \pi^{2}} K \bar{K} \kappa^{4}\left(\frac{\alpha^{\prime}}{2}\right)^{4} \int_{\mathcal{M}_{1}} d^{2} \tau Z_{1}^{10} \prod_{i=2}^{4} \int d^{2} z_{i}\left|N_{(2,1)}\right|^{2}\left\langle\prod_{i=1}^{4} \mathrm{e}^{i k X\left(z_{i}\right)}\right\rangle_{1}
$$

which upon using (2.31),

$$
\left|N_{(2,1)}\right|^{2}\left\langle\prod_{i=1}^{4} \mathrm{e}^{i k X\left(z_{i}\right)}\right\rangle=(2 \pi)^{10} \delta^{(10)}(k) \frac{2^{25} 3^{4} R^{2}}{(2 \pi)^{5} \alpha^{\prime 5}}\left(\frac{\alpha^{\prime}}{2}\right)^{4} \prod_{i<j} F_{1}\left(z_{i}, z_{j}\right)^{\alpha k^{i} \cdot k^{j}}
$$

and $Z_{1}^{10}=\left(2 \tau_{2}\right)^{-5}$ finally becomes

$$
\mathcal{A}_{1}=(2 \pi)^{10} \delta^{(10)}(k) \frac{\kappa^{4} K \bar{K}}{2^{9} \pi^{2} \alpha^{\prime}}\left(\frac{\alpha^{\prime}}{2}\right)^{8} \int_{\mathcal{M}_{1}} \frac{d^{2} \tau}{\tau_{2}^{5}} \prod_{i=2}^{4} \int d^{2} z_{i} \prod_{i<j}^{4} F_{1}\left(z_{i}, z_{j}\right)^{\alpha k^{i} \cdot k^{j}} .
$$

It should be pointed out that the previous computation in [28] claimed that the 1-loop computation in the pure spinor formalism agreed with the RNS result of [33], but it was incorrectly used that $\int d^{2} z w_{1} w_{1} \mu_{\bar{z}}^{z}=2$ instead of $=1$. And to compare with the result of [33] one takes into account the translation invariance of the torus to integrate the "extra" $\int \frac{d^{2} z_{1}}{\tau_{2}}=2$ integral in their equation (2.22) to conclude that (4.14) differs ${ }^{8}$ by $\frac{1}{4}$ from the RNS result reported in [33]. We argue that the one-loop result of [33] is missing the two factors of $1 / 2$ from the GSO projection for both the left- and right-moving sectors, explaining the $1 / 2^{2}$ discrepancy. ${ }^{9}$

\footnotetext{
${ }^{8}$ There is a missing factor of $\left(\alpha^{\prime} / 2\right)^{8}$ in $[33]$.

${ }^{9}$ We thank Eric D'Hoker for kindly confirming to us their missing $1 / 4$ factor [55].
} 


\section{Two-loop}

The two-loop massless four-point amplitude in the non-minimal pure spinor formalism is given by

$$
\mathcal{A}_{2}=\frac{1}{2} \kappa^{4} e^{2 \lambda} \prod_{i=1}^{4} \prod_{j=1}^{3} \int_{\mathcal{M}_{2}} d^{2} \tau_{j} \int d^{2} z_{i}\left\langle\left|\mathcal{N}\left(b, \mu_{j}\right) U^{i}\left(z_{i}\right)\right|^{2}\right\rangle
$$

where \langle\rangle denote the zero-mode integrations $\prod_{I=1}^{2} \int[d \theta]\left[d d^{I}\right][d r]\left[d s^{I}\right]\left[d \bar{w}^{I}\right]\left[d w^{I}\right][d \lambda][d \bar{\lambda}]$ and

$$
\left(b, \mu_{j}\right)=\frac{1}{2 \pi} \int d^{2} y_{j} b_{z z} \mu_{j \bar{z}}^{z}
$$

The 32 (22) zero-modes of $d_{\alpha}\left(s^{\alpha}\right)$ are denoted by $d_{\alpha}^{I}\left(s_{I}^{\alpha}\right)$ for $I=1,2$. As shown in [13], they are saturated by the different factors of (5.1) as

$$
\mathcal{N} \rightarrow\left(s^{1} d^{1}\right)^{11}\left(s^{2} d^{2}\right)^{11} \quad \prod_{j=1}^{3}\left(b, \mu_{j}\right) \rightarrow\left(d^{1}\right)^{3}\left(d^{2}\right)^{3} \quad U^{1} U^{2} U^{3} U^{4} \rightarrow\left(d^{1}\right)^{2}\left(d^{2}\right)^{2},
$$

so that each b-ghost contributes only zero-modes with the term $\left(\frac{\alpha^{\prime}}{2}\right) \frac{\left(\bar{\lambda} \gamma^{m n p} r\right)}{192(\lambda \bar{\lambda})^{2}}\left(d \gamma_{m n p} d\right)$. The expansion $d_{\alpha}\left(y_{i}\right)=\hat{d}_{\alpha}(z)+d_{\alpha}^{1} w_{1}\left(y_{i}\right)+d_{\alpha}^{2} w_{2}\left(y_{i}\right)$ implies a zero-mode contribution of

$$
\left(d \gamma_{m n p} d\right)(y)=\left(d^{1} \gamma_{m n p} d^{1}\right) f_{11}(y)+2\left(d^{1} \gamma_{m n p} d^{2}\right) f_{12}(y)+\left(d^{2} \gamma_{m n p} d^{2}\right) f_{22}(y)
$$

where $f_{i j}(y) \equiv w_{i}(y) w_{j}(y), i, j=1,2$ is the basis of holomorphic quadratic differentials for the genus-2 Riemann surface [56]. It follows from a short computation that,

$$
\begin{aligned}
& \prod_{j=1}^{3}\left(b, \mu_{j}\right)=c_{b} \prod_{j=1}^{3} \int d^{2} y_{j} \mu_{j}\left(y_{j}\right) \Delta\left(y_{1}, y_{2}\right) \Delta\left(y_{2}, y_{3}\right) \Delta\left(y_{3}, y_{1}\right) \\
& \times \frac{1}{(\lambda \bar{\lambda})^{6}}\left(\bar{\lambda} \gamma_{a b c} r\right)\left(\bar{\lambda} \gamma_{d e f} r\right)\left(\bar{\lambda} \gamma_{g h i} r\right)\left(d^{1} \gamma^{a b c} d^{1}\right)\left(d^{1} \gamma^{d e f} d^{2}\right)\left(d^{2} \gamma^{g h i} d^{2}\right)
\end{aligned}
$$

where $c_{b}=\frac{2}{(384 \pi)^{3}}\left(\frac{\alpha^{\prime}}{2}\right)^{3}$ and $\Delta(y, z)=w_{1}(y) w_{2}(z)-w_{2}(y) w_{1}(z)$. In the computation of (5.4) one can check that combinations containing a different number of $d_{\alpha}^{1}$ and $d_{\alpha}^{2}$ zero modes e.g.,

$$
\left(\bar{\lambda} \gamma_{a b c} r\right)\left(\bar{\lambda} \gamma_{d e f} r\right)\left(\bar{\lambda} \gamma_{g h i} r\right)\left(d^{1} \gamma^{a b c} d^{2}\right)\left(d^{1} \gamma^{d e f} d^{2}\right)\left(d^{2} \gamma^{g h i} d^{2}\right)
$$

vanish trivially due to the index symmetries, confirming the zero mode counting of (5.3). Using the period matrix parametrization of moduli space the b-ghost insertions become

$$
\begin{gathered}
\int_{\mathcal{M}_{2}} d^{2} \tau_{1} d^{2} \tau_{2} d^{2} \tau_{3}\left|\prod_{j=1}^{3}\left(b, \mu_{j}\right)\right|^{2}= \\
=c_{b}^{2} \int_{\mathcal{M}_{2}} d^{2} \Omega_{I J}\left|\frac{1}{(\lambda \bar{\lambda})^{6}}\left(\bar{\lambda} \gamma_{a b c} r\right)\left(\bar{\lambda} \gamma_{d e f} r\right)\left(\bar{\lambda} \gamma_{g h i} r\right)\left(d^{1} \gamma^{a b c} d^{1}\right)\left(d^{1} \gamma^{d e f} d^{2}\right)\left(d^{2} \gamma^{g h i} d^{2}\right)\right|^{2}
\end{gathered}
$$

where $\int d^{2} \Omega_{I J}=\int d^{2} \Omega_{11} d^{2} \Omega_{12} d^{2} \Omega_{22}$ and we used the identity of the appendix B. 
The integration over $\left[d w^{I}\right]\left[d \bar{w}^{I}\right]$ can be done using the results of [28] taking into account the different normalizations for the measures (2.11) and (2.12),

$$
\int\left[d w^{1}\right]\left[d \bar{w}^{1}\right]\left[d w^{2}\right]\left[d \bar{w}^{2}\right] e^{-\left(w^{1} \bar{w}^{1}\right)-\left(w^{2} \bar{w}^{2}\right)}=\frac{(\lambda \bar{\lambda})^{6}}{(2 \pi)^{22}} Z_{2}^{-22}
$$

It is straightforward to use the measure $(2.14)$ to integrate over $\left[d s^{1}\right]\left[d s^{2}\right]$, and the amplitude (5.1) becomes

$$
\begin{aligned}
\mathcal{A}_{2}= & \frac{\kappa^{4} e^{2 \lambda}}{2^{56} \pi^{26} 3^{6}(11 ! 5 !)^{4}}\left(\frac{\alpha^{\prime}}{2}\right)^{8} \int_{\mathcal{M}_{2}} d^{2} \Omega_{I J} \mid Z_{2}^{-11} \int[d \theta]\left[d d^{1}\right]\left[d d^{2}\right][d r][d \lambda][d \bar{\lambda}] \\
& \times \frac{\mathrm{e}^{-(\lambda \bar{\lambda})-(r \theta)}}{(\lambda \bar{\lambda})^{6}}\left(\bar{\lambda} \gamma_{a b c} r\right)\left(\bar{\lambda} \gamma_{d e f} r\right)\left(\bar{\lambda} \gamma_{g h i} r\right)\left(d^{1} \gamma^{a b c} d^{1}\right)\left(d^{1} \gamma^{d e f} d^{2}\right)\left(d^{2} \gamma^{g h i} d^{2}\right) \\
& \times\left(\lambda \gamma^{m_{1}}\right)_{\alpha_{1}}\left(\lambda \gamma^{n_{1}}\right)_{\alpha_{2}}\left(\lambda \gamma^{p_{1}}\right)_{\alpha_{3}}\left(\gamma_{m_{1} n_{1} p_{1}}\right)_{\alpha_{4} \alpha_{5}}\left(\lambda \gamma^{m_{2}}\right)_{\beta_{1}}\left(\lambda \gamma^{n_{2}}\right)_{\beta_{2}}\left(\lambda \gamma^{p_{2}}\right)_{\beta_{3}}\left(\gamma_{m_{2} n_{2} p_{2}}\right)_{\beta_{4} \beta_{5}} \\
& \times \epsilon^{\alpha_{1} \ldots \alpha_{5} \rho_{1} \ldots \rho_{11}} \epsilon^{\beta_{1} \ldots \beta_{5} \delta_{1} \ldots \delta_{11}} d_{\rho_{1}}^{1} \ldots d_{\rho_{11}}^{1} d_{\delta_{1}}^{2} \ldots d_{\delta_{11}}^{2} \\
& \times \\
& \left(d^{1} W^{1}\right)\left(d^{1} W^{2}\right)\left(d^{2} W^{3}\right)\left(d^{2} W^{4}\right) w_{1}\left(z_{1}\right) w_{1}\left(z_{2}\right) w_{2}\left(z_{3}\right) w_{2}\left(z_{4}\right) \\
& +\left(d^{1} W^{1}\right)\left(d^{2} W^{2}\right)\left(d^{1} W^{3}\right)\left(d^{2} W^{4}\right) w_{1}\left(z_{1}\right) w_{2}\left(z_{2}\right) w_{1}\left(z_{3}\right) w_{2}\left(z_{4}\right) \\
& +\left(d^{1} W^{1}\right)\left(d^{2} W^{2}\right)\left(d^{2} W^{3}\right)\left(d^{1} W^{4}\right) w_{1}\left(z_{1}\right) w_{2}\left(z_{2}\right) w_{2}\left(z_{3}\right) w_{1}\left(z_{4}\right) \\
& +\left(d^{2} W^{1}\right)\left(d^{2} W^{2}\right)\left(d^{1} W^{3}\right)\left(d^{1} W^{4}\right) w_{2}\left(z_{1}\right) w_{2}\left(z_{2}\right) w_{1}\left(z_{3}\right) w_{1}\left(z_{4}\right) \\
& +\left(d^{2} W^{1}\right)\left(d^{1} W^{2}\right)\left(d^{1} W^{3}\right)\left(d^{2} W^{4}\right) w_{2}\left(z_{1}\right) w_{1}\left(z_{2}\right) w_{1}\left(z_{3}\right) w_{2}\left(z_{4}\right) \\
& \left.+\left(d^{2} W^{1}\right)\left(d^{1} W^{2}\right)\left(d^{2} W^{3}\right)\left(d^{1} W^{4}\right) w_{2}\left(z_{1}\right) w_{1}\left(z_{2}\right) w_{2}\left(z_{3}\right) w_{1}\left(z_{4}\right)\right]\left.\right|^{2} \times\left\langle\prod_{i=1}^{4} e^{i k \cdot x}\right\rangle_{2} \quad(5.6
\end{aligned}
$$

where the only non-vanishing contribution from the external vertices contains two $d^{1}$ and two $d^{2}$ zero-modes coming from $\left(\alpha^{\prime} / 2\right)^{4}(d W)^{4}$. Integrating the $d_{\alpha}$ zero-modes in (5.6) using (2.15) and (4.8)-(4.10) one gets

$$
\mathcal{A}_{2}=\frac{\pi^{6}}{2^{4} 3^{2}}\left(\frac{\alpha^{\prime}}{2}\right)^{6} \int_{\mathcal{M}_{2}} d^{2} \Omega_{I J} Z_{2}^{10}\left|\mathcal{K}_{2}\right|^{2} \times\left\langle\prod_{i=1}^{4} e^{i k \cdot x}\right\rangle_{2}
$$

where the non-minimal kinematic factor $\mathcal{K}$ is given by

$$
\begin{aligned}
\mathcal{K}_{2}=\langle & \left\langle\bar{\lambda} \gamma_{m_{1} n_{1} p_{1}} r\right)\left(\bar{\lambda} \gamma_{\text {def }} r\right)\left(\bar{\lambda} \gamma_{m_{2} n_{2} p_{2}} r\right)\left(\lambda \gamma^{m_{1} \text { defm }} m_{2} \lambda\right) \\
\times & {\left[\left(\lambda \gamma^{n_{1}} W^{1}\right)\left(\lambda \gamma^{p_{1}} W^{2}\right)\left(\lambda \gamma^{n_{2}} W^{3}\right)\left(\lambda \gamma^{p_{2}} W^{4}\right)\left(H_{1234}+H_{3412}\right)\right.} \\
& +\left(\lambda \gamma^{n_{1}} W^{1}\right)\left(\lambda \gamma^{p_{1}} W^{3}\right)\left(\lambda \gamma^{n_{2}} W^{2}\right)\left(\lambda \gamma^{p_{2}} W^{4}\right)\left(H_{1324}+H_{2413}\right) \\
& \left.\left.+\left(\lambda \gamma^{n_{1}} W^{1}\right)\left(\lambda \gamma^{p_{1}} W^{4}\right)\left(\lambda \gamma^{n_{2}} W^{2}\right)\left(\lambda \gamma^{p_{2}} W^{3}\right)\left(H_{1423}+H_{2314}\right)\right]\right\rangle_{(-3,2)}
\end{aligned}
$$

and we defined

$$
H_{i j k l}=w_{1}\left(z_{i}\right) w_{1}\left(z_{j}\right) w_{2}\left(z_{k}\right) w_{2}\left(z_{l}\right) .
$$

In the appendix A we will show that

$$
\mathcal{K}_{2}=2^{12} 3^{3} 5 \mathcal{Y}_{s}\left\langle\left(\lambda A^{1}\right)\left(\lambda \gamma^{m} W^{2}\right)\left(\lambda \gamma^{n} W^{3}\right) \mathcal{F}_{m n}^{4}\right\rangle_{(0,2)}=2^{3} 3 \mathcal{Y}_{s} K\left\langle\left(\lambda^{3} \theta^{5}\right)\right\rangle_{(0,2)}
$$


where the second equality follows from (2.28). Hence (5.7) is given by

$$
\mathcal{A}_{2}=\kappa^{4} e^{2 \lambda} 2^{2} \pi^{6} K \bar{K}\left(\frac{\alpha^{\prime}}{2}\right)^{6} \int_{\mathcal{M}_{2}} d^{2} \Omega_{I J} Z_{2}^{10}\left|\mathcal{Y}_{s}\right|^{2}\left|N_{(0,2)}\right|^{2}\left\langle\prod_{i=1}^{4} e^{i k \cdot x}\right\rangle_{2} .
$$

From the formula $(2.31)$ we get

$$
\left|N_{(0,2)}\right|^{2}\left\langle\prod_{i=1}^{4} e^{i k \cdot x}\right\rangle_{2}=(2 \pi)^{10} \delta^{(10)}(k) \frac{\sqrt{2}}{2^{2} \pi^{6} \alpha^{\prime 5}}\left(\frac{\alpha^{\prime}}{2}\right)^{4} \prod_{i<j} F_{2}\left(z_{i}, z_{j}\right)^{\alpha k^{i} \cdot k^{j}}
$$

which together with $Z_{2}^{10}=2^{-10} \operatorname{det}\left(\operatorname{Im} \Omega_{I J}\right)^{-5}$ implies that

$$
\mathcal{A}_{2}=(2 \pi)^{10} \delta^{(10)}(k) \kappa^{4} e^{2 \lambda} \frac{\sqrt{2} K \bar{K}}{2^{10} \alpha^{\prime 5}}\left(\frac{\alpha^{\prime}}{2}\right)^{10} \int_{\mathcal{M}_{2}} \frac{d^{2} \Omega_{I J}}{\left(\operatorname{det} \operatorname{Im} \Omega_{I J}\right)^{5}} \int_{\Sigma_{4}}\left|\mathcal{Y}_{s}\right|^{2} \prod_{i<j} F_{2}\left(z_{i}, z_{j}\right)^{\alpha k^{i} \cdot k^{j}}
$$

which is the final result for the 2-loop amplitude. ${ }^{10}$ And we have shown that the computation of the whole supersymmetric amplitude including its coefficient is straightforward using the non-minimal pure spinor formalism.

\section{Conclusions}

We used the genus- $g$ measures in the non-minimal pure spinor formalism to find the overall coefficient of the two-loop amplitude and have shown that there are no major differences in carrying out the computations when compared against the analogous calculations for the tree-level and one-loop amplitudes. In fact, this task is significantly simplified by the pure spinor superspace identities of [23] linking the four-point kinematic factors. These observations must be compared against the unsolved difficulties in the RNS formalism, which besides having no explicit computations for the whole supermultiplet has to rely on a factorization procedure to find the two-loop coefficient. Furthermore, we argued that the mismatch of $1 / 16$ found in the two-loop amplitude compared with the result of [33] is due to a missing factor of $1 / 4$ from the GSO projection in their one-loop amplitude.

\section{Acknowledgments}

CRM and HG would like to thank Eric D'Hoker, Nathan Berkovits and Stefan Theisen for discussions. CRM acknowledges support by the Deutsch-Israelische Projektkooperation (DIP H52). HG acknowledges support by FAPESP Ph.D grant 07/54623-8.

\section{A Non-minimal two-loop kinematic factor}

The non-minimal two-loop computation of section 5 leads to the kinematic factor

$$
K=\left\langle\left(\bar{\lambda} \gamma^{a b c} D\right)\left(\bar{\lambda} \gamma^{g h i} D\right)\left(\bar{\lambda} \gamma^{\text {def }} D\right)\left(\lambda \gamma_{a d e f g} \lambda\right)\left[\left(\lambda \gamma^{b} W^{1}\right)\left(\lambda \gamma^{c} W^{2}\right)\left(\lambda \gamma^{h} W^{3}\right)\left(\lambda \gamma^{i} W^{4}\right)\right]\right\rangle_{(-3,2)}
$$

\footnotetext{
${ }^{10}$ The coefficient obtained here is $1 / 16$ times the result reported by [33]. This difference can be accounted for by the missing factor of $1 / 4$ in their 1-loop result which is used as input in their fixing of the 2-loop coefficient through factorization.
} 
In [20] it was shown ${ }^{11}$ that (A.1) is proportional to $\left\langle\left(\lambda \gamma^{m n p q r} \lambda\right)\left(\lambda \gamma^{s} W\right) \mathcal{F}_{m n} \mathcal{F}_{p q} \mathcal{F}_{r s}\right\rangle_{(0,2)}$, the kinematic factor obtained in the minimal pure spinor formalism [17], whose equivalence with the RNS result of [2] was established in $[18,23]$. We will now evaluate all the terms in (A.1) to find the exact coefficient announced in (5.10).

To simplify the covariant computation of (A.1) we use $\left(\bar{\lambda} \gamma^{\text {def }} D\right)\left(\lambda \gamma_{\text {adefg }} \lambda\right)=$ $48(\lambda \bar{\lambda})\left(\lambda \gamma^{a g} D\right)-48\left(\lambda \gamma^{a g} \bar{\lambda}\right)(\lambda D)$ and drop the last term because $\left(\lambda \gamma^{m} W^{I}\right)$ is BRST-closed. And for the same reason we can use $\left(\lambda \gamma^{a} \gamma^{g} D\right)$ instead of $\left(\lambda \gamma^{a g} D\right)$ in the first term. Therefore (A.1) becomes

$$
K=48\left\langle\left(\bar{\lambda} \gamma^{g h i} D\right)\left(\lambda \gamma^{a} \gamma^{g} D\right)\left(\bar{\lambda} \gamma^{a b c} D\right)\left[\left(\lambda \gamma^{b} W^{1}\right)\left(\lambda \gamma^{c} W^{2}\right)\left(\lambda \gamma^{h} W^{3}\right)\left(\lambda \gamma^{i} W^{4}\right)\right]\right\rangle_{(-2,2)} .
$$

The strategy to evaluate and simplify ${ }^{12}$ (A.2) is straightforward due to the identities obeyed by the pure spinor $\lambda^{\alpha}$. One uses the SYM equation of motion for $W^{\alpha}$ in the form of

$$
\begin{aligned}
\left(\bar{\lambda} \gamma^{a b c} D\right)\left(\lambda \gamma^{m} W^{1}\right) & =\frac{1}{4}\left(\lambda \gamma^{m} \gamma^{m_{1} n_{1}} \gamma^{a b c} \bar{\lambda}\right) \mathcal{F}_{m_{1} n_{1}}^{1} \\
\left(\lambda \gamma^{a} \gamma^{g} D\right)\left(\lambda \gamma^{m} W^{2}\right) & =\frac{1}{4}\left(\lambda \gamma^{a g m_{2} n_{2} m} \lambda\right) \mathcal{F}_{m_{2} n_{2}}^{2}
\end{aligned}
$$

and uses gamma matrix identities ${ }^{13}$ in such a way as to get factors which vanish by the pure spinor property of $\left(\lambda \gamma^{m}\right)_{\alpha}\left(\lambda \gamma_{m}\right)_{\beta}=0$. For example, one gets identities like

$$
\left(\lambda \gamma^{b} \gamma^{m_{1} n_{1}} \gamma^{a b c} \bar{\lambda}\right)\left(\lambda \gamma^{a} \gamma^{g} D\right)\left[\mathcal{F}_{m_{1} n_{1}}^{1}\left(\lambda \gamma^{c} W^{2}\right)\right]=48(\lambda \bar{\lambda})\left(\lambda \gamma^{a} \gamma^{g} D\right)\left[\mathcal{F}_{a c}^{1}\left(\lambda \gamma^{c} W^{2}\right)\right]
$$

and

$$
\begin{aligned}
& \mathcal{F}_{r s}^{3}\left(\lambda \gamma^{h} \gamma^{r s} \gamma^{a b c} \bar{\lambda}\right)\left(\lambda \gamma^{a}\right)_{\alpha}\left(\lambda \gamma^{b}\right)_{\beta}\left(\lambda \gamma^{c}\right)_{\gamma}= \\
& =16(\lambda \bar{\lambda})\left(\delta_{b}^{h} \mathcal{F}_{a c}^{3}-\delta_{c}^{h} \mathcal{F}_{a b}^{3}-\delta_{a}^{h} \mathcal{F}_{b c}^{3}\right)\left(\lambda \gamma^{a}\right)_{\alpha}\left(\lambda \gamma^{b}\right)_{\beta}\left(\lambda \gamma^{c}\right)_{\gamma} .
\end{aligned}
$$

Following the above steps (A.2) becomes

$$
\begin{aligned}
K= & 576\left\langle( \overline { \lambda } \gamma ^ { g h i } D ) ( \lambda \gamma ^ { a } \gamma ^ { g } D ) \left[\mathcal{F}_{a b}^{1}\left(\lambda \gamma^{b} W^{2}\right)\left(\lambda \gamma^{h} W^{3}\right)\left(\lambda \gamma^{i} W^{4}\right)\right.\right. \\
& \left.\left.-\frac{1}{3} \mathcal{F}_{a b}^{3}\left(\lambda \gamma^{b} W^{1}\right)\left(\lambda \gamma^{h} W^{2}\right)\left(\lambda \gamma^{i} W^{4}\right)-\frac{1}{3} \mathcal{F}_{a b}^{4}\left(\lambda \gamma^{b} W^{1}\right)\left(\lambda \gamma^{h} W^{2}\right)\left(\lambda \gamma^{i} W^{3}\right)+(1 \leftrightarrow 2)\right]\right\rangle_{(-1,2)} \\
& -192\left\langle\left(\bar{\lambda} \gamma^{g a i} D\right)\left(\lambda \gamma^{a} \gamma^{g} D\right)\left[\mathcal{F}_{b c}^{3}\left(\lambda \gamma^{i} W^{4}\right)\left(\lambda \gamma^{b} W^{1}\right)\left(\lambda \gamma^{c} W^{2}\right)+(3 \leftrightarrow 4)\right]\right\rangle_{(-1,2)} .
\end{aligned}
$$

The last line of (A.7) vanishes. To see this note that the factor inside brackets is BRSTclosed, so that we can replace $\left(\lambda \gamma^{a} \gamma^{g} D\right)$ by $\left(\lambda \gamma^{a g} D\right)$. Furthermore $\left(\bar{\lambda} \gamma^{g a i} D\right)\left(\lambda \gamma^{g a} D\right)=$ $-\left(\bar{\lambda} \gamma^{g a} \gamma^{i} D\right)\left(\lambda \gamma^{g a} D\right)-2\left(\bar{\lambda} \gamma^{a} D\right)\left(\lambda \gamma^{i a} D\right)$ and the last term vanishes when acting on

\footnotetext{
${ }^{11}$ There is a loophole in the proof of [20] though. In that proof the terms in (A.1) which are of the form $k W W W F$ where argued to vanish after summing over the permutations. However we show here that by using the identities of [23] those terms are actually proportional to $W \mathcal{F} \mathcal{F} \mathcal{F}$, so the conclusions of [20] still hold true. CM would like to acknowledge a question made by I. Park which sparked the motivation to revisit that proof.

${ }^{12}$ These kind of computations confirm the observations made long ago that pure spinors simplify the description of super-Yang-Mills theory [57, 58].

${ }^{13}$ The package GAMMA [59] is often very useful for these manipulations.
} 
$\mathcal{F}_{b c}^{3}\left(\lambda \gamma^{i} W^{4}\right)\left(\lambda \gamma^{b} W^{1}\right)\left(\lambda \gamma^{c} W^{2}\right)$ because $\left(\lambda \gamma^{i a} D\right)=\left(\lambda \gamma^{i} \gamma^{a} D\right)-\delta_{a}^{i}(\lambda D)$ and $\left(\lambda \gamma^{i}\right)_{\alpha}\left(\lambda \gamma_{i}\right)_{\beta}=0$ due to the pure spinor property. Therefore by using the gamma matrix identity of

$$
\left(\gamma^{m n}\right)_{\alpha}{ }^{\delta}\left(\gamma_{m n}\right)_{\beta}{ }^{\sigma}=-8 \delta_{\alpha}^{\sigma} \delta_{\beta}^{\delta}-2 \delta_{\alpha}^{\delta} \delta_{\beta}^{\sigma}+4 \gamma_{\alpha \beta}^{m} \gamma_{m}^{\delta \sigma}
$$

and dropping the term proportional to the BRST charge and using momentum conservation (so that $D_{\alpha}$ and $D_{\beta}$ effectively anti-commute) we get

$$
\left(\bar{\lambda} \gamma^{g a} \gamma^{i} D\right)\left(\lambda \gamma^{g a} D\right)=8(\lambda \bar{\lambda})\left(D \gamma^{i} D\right)+4\left(\bar{\lambda} \gamma^{m} D\right)\left(\lambda \gamma^{m} \gamma^{i} D\right)
$$

The first term in the r.h.s. of (A.9) is proportional to $k^{i}$ and vanishes by momentum conservation, while the last term vanishes when acting on $\mathcal{F}_{b c}^{3}\left(\lambda \gamma^{i} W^{4}\right)\left(\lambda \gamma^{b} W^{1}\right)\left(\lambda \gamma^{c} W^{2}\right)$ for the same reason as explained above.

For convenience we write (A.7) as

$$
K=576 K_{a_{1}}-192 K_{a_{2}}-192 K_{a_{3}}+(1 \leftrightarrow 2)
$$

where

$$
K_{a_{1}} \equiv\left\langle\left(\bar{\lambda} \gamma^{g h i} D\right)\left(\lambda \gamma^{a} \gamma^{g} D\right)\left[\mathcal{F}_{a b}^{1}\left(\lambda \gamma^{b} W^{2}\right)\left(\lambda \gamma^{h} W^{3}\right)\left(\lambda \gamma^{i} W^{4}\right)\right]\right\rangle_{(-1,2)}
$$

while $K_{a_{2}}$ and $K_{a_{3}}$ can be obtained by permuting the labels in $K_{a_{1}}$. Using the SYM equations of motion and a few gamma matrix identities we get

$$
\begin{aligned}
K_{a_{1}}= & +\left\langle( \overline { \lambda } \gamma ^ { g h i } D ) \left[ 6 k_{c}^{1}\left(\lambda \gamma^{g} W^{1}\right)\left(\lambda \gamma^{c} W^{2}\right)\left(\lambda \gamma^{h} W^{3}\right)\left(\lambda \gamma^{i} W^{4}\right)\right.\right. \\
& -\frac{1}{4}\left(\lambda \gamma^{m n p q g} \lambda\right) \mathcal{F}_{m n}^{1} \mathcal{F}_{p q}^{2}\left(\lambda \gamma^{h} W^{3}\right)\left(\lambda \gamma^{i} W^{4}\right)-\frac{1}{4}\left(\lambda \gamma^{a g m n h} \lambda\right) \mathcal{F}_{a c}^{1} \mathcal{F}_{m n}^{3}\left(\lambda \gamma^{c} W^{2}\right)\left(\lambda \gamma^{i} W^{4}\right) \\
& \left.\left.-\frac{1}{4}\left(\lambda \gamma^{a g m n h} \lambda\right) \mathcal{F}_{a c}^{1} \mathcal{F}_{m n}^{4}\left(\lambda \gamma^{c} W^{2}\right)\left(\lambda \gamma^{i} W^{3}\right)\right]\right\rangle_{(-1,2)} .
\end{aligned}
$$

After a long and tedious computation using straightforward manipulations and identities like $\left(\lambda \gamma^{m n p q r} \lambda\right) \mathcal{F}_{m n}^{I} \mathcal{F}_{p q}^{J}=\left(\lambda \gamma^{m n p q r} \lambda\right) \mathcal{F}_{m n}^{J} \mathcal{F}_{p q}^{I}$ and [17]

$$
\left(\lambda \gamma^{m n p q r} \lambda\right)\left(\lambda \gamma^{s} W^{4}\right)\left[\mathcal{F}_{m n}^{1} \mathcal{F}_{p q}^{2} \mathcal{F}_{r s}^{3}+\mathcal{F}_{m n}^{3} \mathcal{F}_{p q}^{1} \mathcal{F}_{r s}^{2}+\mathcal{F}_{m n}^{2} \mathcal{F}_{p q}^{3} \mathcal{F}_{r s}^{1}\right]=0
$$

one gets

$$
\begin{aligned}
& K_{a_{1}}=-\frac{1}{2}\left\langle k_{m}^{1}\left(\bar{\lambda} \gamma^{g h i} \gamma^{n} W^{1}\right) \mathcal{F}_{p q}^{2}\left(\lambda \gamma^{m n p q g} \lambda\right)\left(\lambda \gamma^{h} W^{3}\right)\left(\lambda \gamma^{i} W^{4}\right)\right\rangle_{(-1,2)}+(1 \leftrightarrow 2) \\
& -\frac{1}{4}\left\langle\left(2 \mathcal{F}_{r s}^{3} k_{[a}^{1}\left(\bar{\lambda} \gamma^{g h i} \gamma_{c]} W^{1}\right)+2 k_{r}^{3}\left(\bar{\lambda} \gamma^{g h i} \gamma^{s} W^{3}\right) \mathcal{F}_{a c}^{1}\right)\left(\lambda \gamma^{a g r s h} \lambda\right)\left(\lambda \gamma^{c} W^{2}\right)\left(\lambda \gamma^{i} W^{4}\right)\right\rangle_{(-1,2)} \\
& -\frac{1}{4}\left\langle\left(2 \mathcal{F}_{r s}^{4} k_{[a}^{1}\left(\bar{\lambda} \gamma^{g h i} \gamma_{c]} W^{1}\right)+2 k_{r}^{4}\left(\bar{\lambda} \gamma^{g h i} \gamma^{s} W^{4}\right) \mathcal{F}_{a c}^{1}\right)\left(\lambda \gamma^{a g r s h} \lambda\right)\left(\lambda \gamma^{c} W^{2}\right)\left(\lambda \gamma^{i} W^{3}\right)\right\rangle_{(-1,2)} \\
& +\left\langle\left(\lambda \gamma^{m n p q r} \lambda\right)\left[\left(\mathcal{F}_{m n}^{1} \mathcal{F}_{p q}^{3} \mathcal{F}_{r s}^{2}-4 \mathcal{F}_{m n}^{1} \mathcal{F}_{p q}^{2} \mathcal{F}_{r s}^{3}\right)\left(\lambda \gamma^{s} W^{4}\right)-3 \mathcal{F}_{m n}^{3} \mathcal{F}_{p q}^{4} \mathcal{F}_{r s}^{1}\left(\lambda \gamma^{s} W^{2}\right)+(3 \leftrightarrow 4)\right]\right. \\
& -72 k_{m}^{1}\left(\lambda \gamma^{m} W^{2}\right)\left[\mathcal{F}_{h i}^{1}\left(\lambda \gamma^{h} W^{3}\right)\left(\lambda \gamma^{i} W^{4}\right)+\mathcal{F}_{h i}^{3}\left(\lambda \gamma^{h} W^{1}\right)\left(\lambda \gamma^{i} W^{4}\right)+\mathcal{F}_{h i}^{4}\left(\lambda \gamma^{h} W^{1}\right)\left(\lambda \gamma^{i} W^{3}\right)\right] \\
& \left.+24 k_{m}^{1}\left(\lambda \gamma^{m} W^{4}\right) \mathcal{F}_{h i}^{2}\left(\lambda \gamma^{h} W^{1}\right)\left(\lambda \gamma^{i} W^{3}\right)+24 k_{m}^{1}\left(\lambda \gamma^{m} W^{3}\right) \mathcal{F}_{h i}^{2}\left(\lambda \gamma^{h} W^{1}\right)\left(\lambda \gamma^{i} W^{4}\right)\right\rangle_{(0,2)}(\mathrm{A} .13)
\end{aligned}
$$


To simplify the \langle\rangle$_{(-1,2)}$ terms in (A.13) it is convenient to have $\bar{\lambda}_{\alpha}$ in the combination $(\lambda \bar{\lambda})$ by using the identities,

$$
\left(\bar{\lambda} \gamma^{g h i} \gamma^{n} W^{1}\right)\left(\lambda \gamma^{m n p q g} \lambda\right)\left(\lambda \gamma^{h} W^{3}\right)\left(\lambda \gamma^{i} W^{4}\right)=2(\lambda \bar{\lambda})\left(W^{3} \gamma^{g i} \gamma_{n} W^{1}\right)\left(\lambda \gamma^{m n p q g} \lambda\right)\left(\lambda \gamma^{i} W^{4}\right)
$$

and similarly

$$
\begin{aligned}
& \left(\bar{\lambda} \gamma^{g h i} \gamma^{a} W^{1}\right)\left(\lambda \gamma^{a g r s h} \lambda\right)\left(\lambda \gamma^{c} W^{2}\right)\left(\lambda \gamma^{i} W^{4}\right)=2(\lambda \bar{\lambda})\left(W^{4} \gamma^{a h i} W^{1}\right)\left(\lambda \gamma^{a h i r s} \lambda\right)\left(\lambda \gamma^{c} W^{2}\right) \\
& \left(\bar{\lambda} \gamma^{g h i} \gamma^{c} W^{1}\right)\left(\lambda \gamma^{a g r s h} \lambda\right)\left(\lambda \gamma^{c} W^{2}\right)\left(\lambda \gamma^{i} W^{4}\right)=2(\lambda \bar{\lambda})\left(W^{4} \gamma^{g h c} W^{1}\right)\left(\lambda \gamma^{a g r s h} \lambda\right)\left(\lambda \gamma^{c} W^{2}\right) .
\end{aligned}
$$

In $[23]$ it was proved that

$$
\left\langle\left(\lambda \gamma^{m n p q r} \lambda\right)\left(\lambda \gamma^{s} W^{4}\right) \mathcal{F}_{m n}^{1} \mathcal{F}_{p q}^{2} \mathcal{F}_{r s}^{3}\right\rangle_{(n, g)}=-16\left(k^{1} \cdot k^{2}\right)\left\langle\left(\lambda A^{1}\right)\left(\lambda \gamma^{m} W^{2}\right)\left(\lambda \gamma^{n} W^{3}\right) \mathcal{F}_{m n}^{4}\right\rangle_{(n, g)}
$$

and that $\left\langle\left(\lambda A^{1}\right)\left(\lambda \gamma^{m} W^{2}\right)\left(\lambda \gamma^{n} W^{3}\right) \mathcal{F}_{m n}^{4}\right\rangle_{(n, g)}$ is completely symmetric in the particle labels, hence

$$
\begin{gathered}
\left\langle\left(\lambda \gamma^{m n p q r} \lambda\right)\left[\left(\mathcal{F}_{m n}^{1} \mathcal{F}_{p q}^{3} \mathcal{F}_{r s}^{2}-4 \mathcal{F}_{m n}^{1} \mathcal{F}_{p q}^{2} \mathcal{F}_{r s}^{3}\right)\left(\lambda \gamma^{s} W^{4}\right)-3 \mathcal{F}_{m n}^{3} \mathcal{F}_{p q}^{4} \mathcal{F}_{r s}^{1}\left(\lambda \gamma^{s} W^{2}\right)\right]\right\rangle_{(0,2)} \\
+(3 \leftrightarrow 4)=+240\left(k^{1} \cdot k^{2}\right)\left\langle\left(\lambda A^{1}\right)\left(\lambda \gamma^{m} W^{2}\right)\left(\lambda \gamma^{n} W^{3}\right) \mathcal{F}_{m n}^{4}\right\rangle_{(0,2)},
\end{gathered}
$$

where we also used the momentum conservation relation of $\left(k^{1} \cdot k^{3}\right)+\left(k^{1} \cdot k^{4}\right)=-\left(k^{1} \cdot k^{2}\right)$. The last two lines of (A.13) can be simplified by using $\left(\lambda \gamma^{m} W\right)=Q A^{m}-k^{m}(\lambda A)$ and by noticing that the terms of the form $Q\left(A^{m}\right) \mathcal{F}_{p q}\left(\lambda \gamma^{p} W\right)\left(\lambda \gamma^{q} W\right)$ are BRST exact and therefore vanish. Doing that one gets

$$
\begin{gathered}
-72\left\langle k_{m}^{1}\left(\lambda \gamma^{m} W^{2}\right)\left[\mathcal{F}_{h i}^{1}\left(\lambda \gamma^{h} W^{3}\right)\left(\lambda \gamma^{i} W^{4}\right)+\mathcal{F}_{h i}^{3}\left(\lambda \gamma^{h} W^{1}\right)\left(\lambda \gamma^{i} W^{4}\right)+\mathcal{F}_{h i}^{4}\left(\lambda \gamma^{h} W^{1}\right)\left(\lambda \gamma^{i} W^{3}\right)\right]\right. \\
\left.+24 k_{m}^{1}\left(\lambda \gamma^{m} W^{4}\right) \mathcal{F}_{h i}^{2}\left(\lambda \gamma^{h} W^{1}\right)\left(\lambda \gamma^{i} W^{3}\right)+24 k_{m}^{1}\left(\lambda \gamma^{m} W^{3}\right) \mathcal{F}_{h i}^{2}\left(\lambda \gamma^{h} W^{1}\right)\left(\lambda \gamma^{i} W^{4}\right)\right\rangle_{(0,2)} \\
=+240\left(k^{1} \cdot k^{2}\right)\left\langle\left(\lambda A^{1}\right)\left(\lambda \gamma^{m} W^{2}\right)\left(\lambda \gamma^{n} W^{3}\right) \mathcal{F}_{m n}^{4}\right\rangle_{(0,2)} .
\end{gathered}
$$

Feeding the results above into the expression for $K_{a_{1}}$ in (A.13) one can write it as $K_{a_{1}}=K_{a_{11}}+K_{a_{12}}$, where

$$
\begin{aligned}
K_{a_{11}}= & -\left\langle k_{r}^{1}\left(\lambda \gamma^{m n p q r} \lambda\right)\left(W^{3} \gamma_{m n s} W^{1}\right)\left(\lambda \gamma^{s} W^{4}\right) \mathcal{F}_{p q}^{2}\right\rangle_{(0,2)}+(1 \leftrightarrow 2) \\
& -\left[\left\langle\left(\mathcal{F}_{r s}^{3} k_{[a}^{1}\left(W^{4} \gamma_{g h \mid c]} W^{1}\right)+k_{r}^{3}\left(W^{4} \gamma_{g h s} W^{3}\right) \mathcal{F}_{a c}^{1}\right)\left(\lambda \gamma^{a g r s h} \lambda\right)\left(\lambda \gamma^{c} W^{2}\right)\right\rangle_{(0,2)}+(3 \leftrightarrow 4)\right]
\end{aligned}
$$

and

$$
K_{a_{12}}=+480\left(k^{1} \cdot k^{2}\right)\left\langle\left(\lambda A^{1}\right)\left(\lambda \gamma^{m} W^{2}\right)\left(\lambda \gamma^{n} W^{3}\right) \mathcal{F}_{m n}^{4}\right\rangle_{(0,2)}
$$

Furthermore, by using the gamma matrix identities $\gamma^{m n p}=\gamma^{m n} \gamma^{p}-\eta^{m n} \gamma^{p}+\eta^{a m} \gamma^{n}$ and

$$
\left(\gamma^{m n}\right)_{\alpha}{ }^{\delta}\left(\gamma_{m n}\right)_{\beta}{ }^{\sigma}=-8 \delta_{\alpha}^{\sigma} \delta_{\beta}^{\delta}+4 \gamma_{\alpha \beta}^{m} \gamma_{m}^{\delta \sigma}-2 \delta_{\alpha}^{\delta} \delta_{\beta}^{\sigma},
$$

the pure spinor identities $\left(\lambda \gamma^{a m n p q} \lambda\right)\left(\lambda \gamma_{a}\right)_{\beta}=\left(\lambda \gamma^{m}\right)_{\alpha}\left(\lambda \gamma_{m}\right)_{\beta}=0$, the equation of motion $k_{m}^{I}\left(\lambda \gamma^{m} W^{I}\right)=0$ and the results above, $K_{a_{11}}$ (and its permutations $K_{a_{21}}$ and $K_{a_{31}}$ ) can be further simplified. In fact, one can show that

$$
-\left\langle k_{r}^{1}\left(\lambda \gamma^{m n p q r} \lambda\right)\left(W^{3} \gamma_{m n s} W^{1}\right)\left(\lambda \gamma^{s} W^{4}\right) \mathcal{F}_{p q}^{2}\right\rangle_{(0,2)}
$$




$$
\begin{aligned}
& =32\left\langle k_{m}^{1}\left(\lambda \gamma^{m} W^{4}\right)\left(\lambda \gamma^{p} W^{3}\right)\left(\lambda \gamma^{q} W^{1}\right) \mathcal{F}_{p q}^{2}\right\rangle_{(0,2)}+(3 \leftrightarrow 4) \\
& =-32\left(\left(k^{1} \cdot k^{3}\right)+\left(k^{1} \cdot k^{4}\right)\right)\left\langle\left(\lambda A^{1}\right)\left(\lambda \gamma^{m} W^{2}\right)\left(\lambda \gamma^{n} W^{3}\right) \mathcal{F}_{m n}^{4}\right\rangle_{(0,2)} .
\end{aligned}
$$

From $\gamma_{\alpha \beta}^{m n p} \gamma_{m n p}^{\gamma \delta}=48\left(\delta_{\alpha}^{\gamma} \delta_{\beta}^{\delta}-\delta_{\alpha}^{\delta} \delta_{\beta}^{\gamma}\right)$ and the equation of motion for $W_{3}^{\alpha}$ it follows that,

$$
-k_{r}^{3}\left(\lambda \gamma^{a g r s h} \lambda\right)\left(W^{4} \gamma_{g h s} W^{3}\right) \mathcal{F}_{a c}^{1}\left(\lambda \gamma^{c} W^{2}\right)=48\left(k^{3} \cdot k^{4}\right)\left(\lambda A^{1}\right)\left(\lambda \gamma^{m} W^{2}\right)\left(\lambda \gamma^{n} W^{3}\right) \mathcal{F}_{m n}^{4}
$$

and

$$
\frac{1}{2} \mathcal{F}_{r s}^{3} k_{c}^{1}\left(W^{4} \gamma_{g h a} W^{1}\right)\left(\lambda \gamma^{a g r s h} \lambda\right)\left(\lambda \gamma^{c} W^{2}\right)=48\left(k^{1} \cdot k^{2}\right)\left(\lambda A^{1}\right)\left(\lambda \gamma^{m} W^{2}\right)\left(\lambda \gamma^{n} W^{3}\right) \mathcal{F}_{m n}^{4} .
$$

From (A.21) one also gets

$$
-\frac{1}{2} \mathcal{F}_{r s}^{3} k_{a}^{1}\left(W^{4} \gamma_{g h c} W^{1}\right)\left(\lambda \gamma^{a g r s h} \lambda\right)\left(\lambda \gamma^{c} W^{2}\right)=16\left(k^{1} \cdot k^{3}\right)\left(\lambda A^{1}\right)\left(\lambda \gamma^{m} W^{2}\right)\left(\lambda \gamma^{n} W^{3}\right) \mathcal{F}_{m n}^{4} .
$$

Plugging the identities (A.21)-(A.22) in (A.19) and summing over the indicated permutations leads to

$$
K_{a_{11}}=240\left(k^{1} \cdot k^{2}\right)\left\langle\left(\lambda A^{1}\right)\left(\lambda \gamma^{m} W^{2}\right)\left(\lambda \gamma^{n} W^{3}\right) \mathcal{F}_{m n}^{4}\right\rangle_{(0,2)}
$$

hence

$$
K_{a_{1}}=K_{a_{11}}+K_{a_{12}}=720\left(k^{1} \cdot k^{2}\right)\left\langle\left(\lambda A^{1}\right)\left(\lambda \gamma^{m} W^{2}\right)\left(\lambda \gamma^{n} W^{3}\right) \mathcal{F}_{m n}^{4}\right\rangle_{(0,2)} .
$$

From (A.10) and (A.24) and their permutations one arrives at the final result ${ }^{14}$ for (A.1),

$$
\begin{aligned}
K= & +720\left\langle\left(\lambda A^{1}\right)\left(\lambda \gamma^{m} W^{2}\right)\left(\lambda \gamma^{n} W^{3}\right) \mathcal{F}_{m n}^{4}\right\rangle_{(0,2)} \times \\
& \times\left[576\left(k^{1} \cdot k^{2}\right)-192\left(k^{3} \cdot k^{2}\right)-192\left(k^{4} \cdot k^{1}\right)+576\left(k^{2} \cdot k^{1}\right)-192\left(k^{3} \cdot k^{1}\right)-192\left(k^{4} \cdot k^{2}\right)\right] \\
= & 3 \cdot 2^{7} \cdot 2880\left(k^{1} \cdot k^{2}\right)\left\langle\left(\lambda A^{1}\right)\left(\lambda \gamma^{m} W^{2}\right)\left(\lambda \gamma^{n} W^{3}\right) \mathcal{F}_{m n}^{4}\right\rangle_{(0,2)} .
\end{aligned}
$$

The complete kinematic factor (5.8) is obtained using the result (A.25) and permuting its labels. The first line of (5.8) is given by (A.25) while the second and third are obtained by replacing $s \rightarrow u$ and $s \rightarrow t$ respectively. The final result is therefore

$$
\begin{aligned}
\mathcal{K}_{2}= & -3 \cdot 2^{6} \cdot 2880\left\langle\left(\lambda A^{1}\right)\left(\lambda \gamma^{m} W^{2}\right)\left(\lambda \gamma^{n} W^{3}\right) \mathcal{F}_{m n}^{4}\right\rangle_{(0,2)} \\
& \times\left[s\left(H_{1234}+H_{3412}\right)+u\left(H_{1324}+H_{2413}\right)+t\left(H_{1423}+H_{2314}\right)\right] \\
= & 2^{12} 3^{3} 5 \mathcal{Y}_{s}\left\langle\left(\lambda A^{1}\right)\left(\lambda \gamma^{m} W^{2}\right)\left(\lambda \gamma^{n} W^{3}\right) \mathcal{F}_{m n}^{4}\right\rangle_{(0,2)}
\end{aligned}
$$

where we used the Mandelstam variables and $u=-t-s$ together with

$$
\begin{aligned}
& H_{1234}+H_{3412}-H_{1324}-H_{2413}=\Delta(1,4) \Delta(2,3) \\
& H_{1423}+H_{2314}-H_{1324}-H_{2413}=-\Delta(1,2) \Delta(3,4) .
\end{aligned}
$$

and the definition (2.38). With (A.26) the expression for the kinematic factor (5.8) is finally demonstrated.

\footnotetext{
${ }^{14}$ To check results we performed explicit component expansion computations with especially-crafted programs using FORM [60, 61].
} 


\section{B Period matrix parametrization of genus-two moduli space}

Let $\mu_{i}^{z} \bar{z}(i=1,2,3)$ be the Beltrami differentials, $\tau_{i}(i=1,2,3)$ the Teichmüller parameters and $w_{I}(z)(I=1,2)$ the holomorphic 1- forms over $\Sigma_{2}$, then [17]

$$
\int d^{2} \tau_{1} d^{2} \tau_{2} d^{2} \tau_{3}\left|\prod_{i=1}^{3} \int d^{2} z_{i} \mu_{i}\left(z_{i}\right) \Delta(1,2) \Delta(2,3) \Delta(3,1)\right|^{2}=\int d^{2} \Omega_{11} d^{2} \Omega_{12} d^{2} \Omega_{22}
$$

where $\Delta(i, j)=w_{1}\left(z_{i}\right) w_{2}\left(z_{j}\right)-w_{1}\left(z_{j}\right) w_{2}\left(z_{i}\right)$. To prove this one uses the identity ${ }^{15}[1,65]$

$$
\int d^{2} z w_{I}(z) w_{J}(z) \mu_{i}(z)=\frac{\delta \Omega_{I J}}{\delta \tau_{i}}
$$

and expands $\Delta(1,2) \Delta(2,3) \Delta(3,1)$ to get

$$
\prod_{i=1}^{3} \int d^{2} z_{i} \mu_{i}\left(z_{i}\right) \Delta(1,2) \Delta(2,3) \Delta(3,1)=-\frac{\delta \Omega_{11}}{\delta \tau_{i}} \frac{\delta \Omega_{12}}{\delta \tau_{j}} \frac{\delta \Omega_{22}}{\delta \tau_{k}} \epsilon^{i j k}
$$

So

$$
\begin{aligned}
d \tau_{1} \wedge d \tau_{2} \wedge d \tau_{3} \prod_{i=1}^{3} \int d^{2} z_{i} \mu_{i}\left(z_{i}\right) \Delta(1,2) \Delta(2,3) \Delta(3,1) & =-\frac{\delta \Omega_{11}}{\delta \tau_{i}} \frac{\delta \Omega_{12}}{\delta \tau_{j}} \frac{\delta \Omega_{22}}{\delta \tau_{k}} \epsilon^{i j k} d \tau_{1} \wedge d \tau_{2} \wedge d \tau_{3} \\
& =-\frac{\delta \Omega_{11}}{\delta \tau_{i}} \frac{\delta \Omega_{12}}{\delta \tau_{j}} \frac{\delta \Omega_{22}}{\delta \tau_{k}} d \tau_{i} \wedge d \tau_{j} \wedge d \tau_{k} \\
& =-\delta \Omega_{11} \wedge \delta \Omega_{12} \wedge \delta \Omega_{22} .
\end{aligned}
$$

Multiplying the last expression by its complex conjugate we get (B.1).

Open Access. This article is distributed under the terms of the Creative Commons Attribution Noncommercial License which permits any noncommercial use, distribution, and reproduction in any medium, provided the original author(s) and source are credited.

\section{References}

[1] E. D'Hoker and D.H. Phong, The geometry of string perturbation theory, Rev. Mod. Phys. 60 (1988) 917 [SPIRES].

[2] E. D'Hoker and D.H. Phong, Two-loop superstrings VI: non-renormalization theorems and the 4-point function, Nucl. Phys. B 715 (2005) 3 [hep-th/0501197] [SPIRES].

[3] R. Medina, F.T. Brandt and F.R. Machado, The open superstring 5-point amplitude revisited, JHEP 07 (2002) 071 [hep-th/0208121] [SPIRES].

[4] L.A. Barreiro and R. Medina, 5-field terms in the open superstring effective action, JHEP 03 (2005) 055 [hep-th/0503182] [SPIRES].

[5] D. Oprisa and S. Stieberger, Six gluon open superstring disk amplitude, multiple hypergeometric series and Euler-Zagier sums, hep-th/0509042 [SPIRES].

\footnotetext{
${ }^{15}$ In the Mathematics literature this is the "Rauch variational formula", see e.g. [62-64].
} 
[6] S. Stieberger, Open 83 closed vs. pure open string disk amplitudes, arXiv:0907.2211 [SPIRES].

[7] D.M. Richards, The one-loop five-graviton amplitude and the effective action, JHEP 10 (2008) 042 [arXiv:0807.2421] [SPIRES].

[8] D.M. Richards, The one-loop $H^{2} R^{3}$ and $H^{2}(D H)^{2} R$ terms in the effective action, JHEP 10 (2008) 043 [arXiv:0807.3453] [SPIRES].

[9] A. Basu, The $D^{1} 0 R^{4}$ term in type IIB string theory, Phys. Lett. B 648 (2007) 378 [hep-th/0610335] [SPIRES].

[10] M.B. Green, J.G. Russo and P. Vanhove, Low energy expansion of the four-particle genus-one amplitude in type-II superstring theory, JHEP 02 (2008) 020 [arXiv:0801.0322] [SPIRES].

[11] N. Berkovits, Super-Poincaré covariant quantization of the superstring, JHEP 04 (2000) 018 [hep-th/0001035] [SPIRES].

[12] N. Berkovits, Multiloop amplitudes and vanishing theorems using the pure spinor formalism for the superstring, JHEP 09 (2004) 047 [hep-th/0406055] [SPIRES].

[13] N. Berkovits, Pure spinor formalism as an $N=2$ topological string, JHEP 10 (2005) 089 [hep-th/0509120] [SPIRES].

[14] N. Berkovits, Explaining the pure spinor formalism for the superstring, JHEP 01 (2008) 065 [arXiv:0712.0324] [SPIRES].

[15] N. Berkovits, ICTP lectures on covariant quantization of the superstring, hep-th/0209059 [SPIRES].

[16] O.A. Bedoya and N. Berkovits, GGI lectures on the pure spinor formalism of the superstring, arXiv:0910.2254 [SPIRES].

[17] N. Berkovits, Super-Poincaré covariant two-loop superstring amplitudes, JHEP 01 (2006) 005 [hep-th/0503197] [SPIRES].

[18] N. Berkovits and C.R. Mafra, Equivalence of two-loop superstring amplitudes in the pure spinor and RNS formalisms, Phys. Rev. Lett. 96 (2006) 011602 [hep-th/0509234] [SPIRES].

[19] G. Policastro and D. Tsimpis, $R^{4}$, purified, Class. Quant. Grav. 23 (2006) 4753 [hep-th/0603165] [SPIRES].

[20] N. Berkovits and C.R. Mafra, Some superstring amplitude computations with the non-minimal pure spinor formalism, JHEP 11 (2006) 079 [hep-th/0607187] [SPIRES].

[21] C.R. Mafra and C. Stahn, The one-loop open superstring massless five-point amplitude with the non-minimal pure spinor formalism, JHEP 03 (2009) 126 [arXiv:0902.1539] [SPIRES].

[22] C.R. Mafra, Simplifying the tree-level superstring massless five-point amplitude, JHEP 01 (2010) 007 [arXiv:0909.5206] [SPIRES].

[23] C.R. Mafra, Pure spinor superspace identities for massless four-point kinematic factors, JHEP 04 (2008) 093 [arXiv: 0801.0580] [SPIRES].

[24] N. Berkovits, Explaining pure spinor superspace, hep-th/0612021 [SPIRES].

[25] N. Berkovits and N. Nekrasov, Multiloop superstring amplitudes from non-minimal pure spinor formalism, JHEP 12 (2006) 029 [hep-th/0609012] [SPIRES].

[26] Y. Aisaka and N. Berkovits, Pure spinor vertex operators in Siegel gauge and loop amplitude regularization, JHEP 07 (2009) 062 [arXiv: 0903.3443] [SPIRES]. 
[27] P.A. Grassi and P. Vanhove, Higher-loop amplitudes in the non-minimal pure spinor formalism, JHEP 05 (2009) 089 [arXiv:0903.3903] [SPIRES].

[28] H. Gomez, One-loop superstring amplitude from integrals on pure spinors space, JHEP 12 (2009) 034 [arXiv:0910.3405] [SPIRES].

[29] M.B. Green, H.-H. Kwon and P. Vanhove, Two loops in eleven dimensions, Phys. Rev. D 61 (2000) 104010 [hep-th/9910055] [SPIRES].

[30] N. Berkovits, New higher-derivative $R^{4}$ theorems, Phys. Rev. Lett. 98 (2007) 211601 [hep-th/0609006] [SPIRES].

[31] E. D'Hoker and D.H. Phong, Lectures on two-loop superstrings, hep-th/0211111 [SPIRES].

[32] A. Morozov, NSR superstring measures revisited, JHEP 05 (2008) 086 [arXiv:0804.3167] [SPIRES].

[33] E. D'Hoker, M. Gutperle and D.H. Phong, Two-loop superstrings and S-duality, Nucl. Phys. B 722 (2005) 81 [hep-th/0503180] [SPIRES].

[34] E.P. Verlinde and H.L. Verlinde, Chiral bosonization, determinants and the string partition function, Nucl. Phys. B 288 (1987) 357 [SPIRES].

[35] E. D'Hoker and D.H. Phong, Two-loop superstrings V: gauge slice independence of the N-point function, Nucl. Phys. B 715 (2005) 91 [hep-th/0501196] [SPIRES].

[36] E. D'Hoker and D.H. Phong, Two-loop superstrings IV, the cosmological constant and modular forms, Nucl. Phys. B 639 (2002) 129 [hep-th/0111040] [SPIRES].

[37] E. D'Hoker and D.H. Phong, Two-loop superstrings III, slice independence and absence of ambiguities, Nucl. Phys. B 636 (2002) 61 [hep-th/0111016] [SPIRES].

[38] E. D'Hoker and D.H. Phong, Two-loop superstrings II, the chiral measure on moduli space, Nucl. Phys. B 636 (2002) 3 [hep-th/0110283] [SPIRES].

[39] E. D'Hoker and D.H. Phong, Two-loop superstrings I, main formulas, Phys. Lett. B 529 (2002) 241 [hep-th/0110247] [SPIRES].

[40] C.R. Mafra, Superstring scattering amplitudes with the pure spinor formalism, arXiv:0902.1552 [SPIRES].

[41] I. Oda and M. Tonin, $Y$-formalism and $b$ ghost in the non-minimal pure spinor formalism of superstrings, Nucl. Phys. B $\mathbf{7 7 9}$ (2007) 63 [arXiv:0704.1219] [SPIRES].

[42] E. Witten, Twistor-like transform in ten-dimensions, Nucl. Phys. B 266 (1986) 245 [SPIRES].

[43] W. Siegel, Superfields in higher dimensional space-time, Phys. Lett. B 80 (1979) 220 [SPIRES].

[44] J.P. Harnad and S. Shnider, Constraints and field equations for ten-dimensional super Yang-Mills theory, Commun. Math. Phys. 106 (1986) 183 [SPIRES].

[45] H. Ooguri, J. Rahmfeld, H. Robins and J. Tannenhauser, Holography in superspace, JHEP 07 (2000) 045 [hep-th/0007104] [SPIRES].

[46] P.A. Grassi and L. Tamassia, Vertex operators for closed superstrings, JHEP 07 (2004) 071 [hep-th/0405072] [SPIRES].

[47] S. Weinberg, Coupling constants and vertex functions in string theories, Phys. Lett. B 156 (1985) 309 [SPIRES]. 
[48] S. Weinberg, Covariant path integral approach to string theory, UTTG-17-87 [SPIRES].

[49] M. Bershadsky, S. Cecotti, H. Ooguri and C. Vafa, Kodaira-Spencer theory of gravity and exact results for quantum string amplitudes, Commun. Math. Phys. 165 (1994) 311 [hep-th/9309140] [SPIRES].

[50] J. Polchinski, String theory. Volume 1: an introduction to the bosonic string, Cambridge University Press, Cambridge U.K. (1998).

[51] J. Polchinski, Factorization of bosonic string amplitudes, Nucl. Phys. B 307 (1988) 61 [SPIRES].

[52] P. Griffiths and J. Harris, Principles of algebraic geometry, Wiley Classics Library Edition Published, U.S.A. (1994).

[53] A.A. Beilinson and Y.I. Manin, The Mumford form and the Polyakov measure in string theory, Commun. Math. Phys. 107 (1986) 359 [SPIRES].

[54] W.I. Weisberger, Normalization of the path integral measure and the coupling constants for bosonic strings, Nucl. Phys. B 284 (1987) 171 [SPIRES].

[55] E. D'Hoker, private communication.

[56] H.M. Farkas and I. Kra, Riemann surfaces, second edition, Springer-Verlag, New York U.S.A. (1992).

[57] P.S. Howe, Pure spinors lines in superspace and ten-dimensional supersymmetric theories, Phys. Lett. B 258 (1991) 141 [Addendum ibid. B 259 (1991) 511] [SPIRES].

[58] P.S. Howe, Pure spinors, function superspaces and supergravity theories in ten-dimensions and eleven-dimensions, Phys. Lett. B 273 (1991) 90 [SPIRES].

[59] U. Gran, GAMMA: a Mathematica package for performing gamma-matrix algebra and Fierz transformations in arbitrary dimensions, hep-th/0105086 [SPIRES].

[60] J.A.M. Vermaseren, New features of FORM, math-ph/0010025 [SPIRES].

[61] M. Tentyukov and J.A.M. Vermaseren, The multithreaded version of FORM, hep-ph/0702279 [SPIRES].

[62] I. Chavel and H.M. Farkas, Differential geometry and complex analysis: a volume dedicated to the memory of Harry Ernest Rauch, Springer-Verlag, Berlin Heidelberg Germany (1985).

[63] S. Nag, The complex analytic theory of Teichmüller spaces, John Wiley \& Sons, U.S.A. (1988).

[64] H.E. Rauch, On the transcendental moduli of algebraic Riemann surfaces, Proc. Natl. Acad. Sci. U.S.A. 41 (1955) 42.

[65] A.A. Belavin, V. Knizhnik, A. Morozov and A. Perelomov, Two and three loop amplitudes in the bosonic string theory, JETP Lett. 43 (1986) 411 [Phys. Lett. B 177 (1986) 324] [SPIRES]. 